\title{
CAUSATION
}

CAUSATION, 16(11): 5-57

DOI:10.5281/zenodo.5746415

Received: November 28, 2021,

Accepted: December 1, 2021

Published: December 1, 2021

ISSN: 1863-9542

\section{Mutually exclusive events}

\section{Research article}

\section{Ilija Barukčić ${ }^{1}$}

${ }^{1}$ Internist, Horandstrasse, 26441 Jever, Germany

* Correspondence: E-Mail: Barukcic@ $@$ t-online.de; Tel: +49-4466-333; Fax: +49-4466-333.

\section{Abstract: \\ Background:}

Why should and how could the occurrence of an event $A_{t}$ at a certain (period of) time / Bernoulli trial $t$ exclude the occurrence of another event $B_{t}$ at the same (period of) time / Bernoulli trial $t$ and vice versa? Can this be described somehow mathematically?

\section{Methods:}

Basic methods of classical logic, probability theory and statistics were used to analyse the interior logic of an exclusion relationship.

\section{Results:}

Mutually exclusive events are mathematized, the relationship to the relative risk and the odds' ratio is worked out.

\section{Conclusion:}

Mathematically, it is possible to recognize mutually exclusive events.

\section{Keywords: Exclusion; Study design; Bias; Cause; Effect; Causal relationship k}

\section{Introduction}

What is going to keep us moving while facing circumstances which forces us more and more to succumb to difficulties? Nowadays, it cannot be overlooked that under the extremely high pressure of the non-ending severe acute respiratory syndrome coronavirus 2 (SARS-CoV-2) pandemics not only human diamonds, but heroes are formed. Even if our world is changing including us humans too, we 
humans are still far from finished. "We are not now that strength which in old days Moved earth and heaven; that which we are, we are; One equal temper of heroic hearts, Made weak by time and fate, but strong in will To strive, to seek, to find, and not to yield "(see Lord Tennyson, 1842, Ulysses, p. 91). Indeed, at the end we are what we are. But after all, because we are what we are,

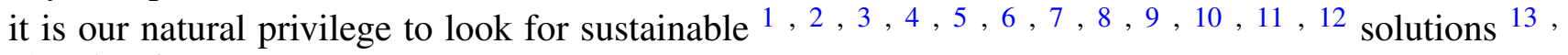
$14,15,16,17,18$ while eye in eye with a threat, who backs away, exposes himself to the danger of getting stuck on the track. This historical crisis and natural disaster, contains in itself the germ of the new and the solution needed. In our human endeavours, to bring a great danger quickly under control, we can rely on logic, probability theory and mathematics too. In logic and probability theory, there are events which are mutually exclusive or disjoint. In other words, there are circumstances, for whatever reason, where i. e. two events cannot both occur at the same time. A example in the extreme is the coin-tossing example. However, being pregnant and being a male human being is another example. As known, it is not possible for a men to be pregnant. Being pregnant and being a men are excluding each other. As known, under normal circumstances, the sample space is a collection or a set of all possible outcomes of an experiment. However, what if emptiness, nothingness or no outcome is the outcome? Can we identify somehow which event and to what extent does an event excludes at the same time the occurrence of a Covid - 19 infection or Covid-19 death?

\section{Material and methods}

\subsection{Data}

\subsection{Study design}

A study design should be fair and representative as much as possible in order to assure data, which we can rely on and work with. The index of unfairness(Barukčić, 2019b) and the index of independence(Barukčić, 2019a) are indicating to which extent data could be biased due to study design. Under ideal conditions, it is desirable that $\mathrm{p}(\mathrm{IOU})=0$ or $\mathrm{p}(\mathrm{IOI})=0$ or that even $\mathrm{p}(\mathrm{IOU})=\mathrm{p}(\mathrm{IOI})=0$.

\footnotetext{
${ }^{1}$ Sputnik V, Russia

${ }^{2}$ AZD1222, AstraZeneca/University Oxford, Sweden/Great Britain

${ }^{3}$ Ad26.COV2.S, Janssen-Cilag International NV

${ }^{4}$ BNT162b2 (Comirnaty ${ }^{\mathrm{TM}}$ ), BioNTech/Pfizer, Germany

${ }^{5}$ Spikevax ${ }^{\mathrm{TM}}$, Moderna Biotech Spain, S.L

${ }^{6}$ DNA SARS-CoV-2 vaccine (ZyCoV-D), India

${ }^{7}$ NVX-CoV2373 Covid-19 Vaccine (PREVENT-19 trial)

${ }^{8}$ Valneva's vaccine

${ }^{9}$ Soberana, Cuba

${ }^{10}$ and other vaccines

${ }^{11}$ Molnupiravir (LAGEVRIO ${ }^{\mathrm{TM}}$ )

${ }^{12}$ Ritonavir (PF-07321332), PAXLOVID ${ }^{\mathrm{TM}}$

${ }^{13}$ Regdanvimab (Regkirona ${ }^{\mathrm{TM}}$ )

${ }^{14}$ Casirivimab and Imdevimab (Ronapreve ${ }^{\mathrm{TM}}$ )

${ }^{15}$ Bamlanivimab

${ }^{16}$ Etesevimab

${ }^{17}$ Sotrovimab

${ }^{18}$ Tixgevimab and Cilgavimab (AZD7442)
} 
Table 1. The quality of data (see Barukčić, 2019b, p. 25)

\begin{tabular}{|c|c|}
\hline $\mathrm{p}(\mathrm{IOI})$ & Quality of study design \\
\hline $0<p(I O I) \leq 0,25$ & Unfair study design \\
$0,25<p(I O I) \leq 0,5$ & Very unfair study design \\
$0,5<p(I O I) \leq 0,75$ & Highly unfair study design \\
$0,75<p(I O I) \leq 1,0$ & Extremely unfair study design \\
\hline
\end{tabular}

\subsubsection{Causality and induction}

Yet ever since thousands of years the topic of causality has been subject to intense controversy. Nonetheless, causality is still a central concept of any philosophy and of any science as a whole. At this point it is desirable but impossible to go into the details of David Hume's famous sceptical view on the relationship between causality and induction (David Hume, A Treatise of Human Nature, 1739, Book 1, part iii, section 6) and Kant's response to Hume's view in his Prolegomena to Any Future Metaphysics (1783). Anyway, the quality of data has influence on the quality of the conclusion drawn. Decisions should be based on high quality of data. However, we are continually asking ourselves can we and to which extent are we allowed to rely on the data published. Data integrity issues in Pfizer's vaccine trial ${ }^{19}$ have become public. Poor practices at clinical trials of different kind ${ }^{20}$ raise questions about the quality of data published and can be suitable to diminish the public trust in a safe and effective Covid-19 vaccine. It is unavoidable that the data provided to the public have to be treated with some caution. However, it has to be ascertained that the quality of the data will be further improved. Besides of these improvements, data should be made freely publicly available in detail while following a certain standard. There are accurate mathematical methods to check data for integrity, while few of them are already discussed ${ }^{21}$ in the literature.

\footnotetext{
${ }^{19}$ Paul D Thacker, investigative journalist PMID: 34728500

${ }^{20}$ The British Medical Journal PMID: 34728500

${ }^{21}$ Hong Chen et al. PMID: 25087521
} 
2.2.2. The study of Grange et al.

Vaccines are effective in preventing COVID-19 deaths. Grange et al. (see Grange et al., 2021) investigated COVID-19-related deaths in 3273336 individuals in Scotland who were fully vaccinated by Aug 18, 2021. Scotland's 2011 census population has been 5313 600. Grange et al. are writing: "Of the 3273336 individuals in Scotland who were fully vaccinated by Aug 18, 2021 (73.6\% of the eligible population), 1205642 individuals received two doses of BNT162b2 and 2026198 individuals received two doses of ChAdOx $1 \mathrm{nCoV}-19$. As there were no deaths among the 41496 individuals who received two doses of mRNA-1273 (Moderna) vaccine during the study period, they were not further considered in this analysis.

236 deaths in fully vaccinated people were recorded (0.007\% of the total vaccinated): $47(0.004 \%)$ of those individuals had received BNT162b2 (median age 74.0 years [IQR 69.0-89.0]), and 188 (0.009\%) individuals had received ChAdOx1 nCoV-19 (80.0 years [73.0-86.0]).'(see Grange et al., 2021).

What is the true placebo group in order to analyse the efficacy of a certain vaccine? Compared one vaccine with other vaccines might lead to bias. In other words, it is appropriate to proof what happens if people are not vaccinated. In the group of non-vaccinated the death rate due to Covid-19 is about 2 per cent. 
2.2.3. Moderna

Table 2. Moderna vaccine and Covid-19 death (Study Grange et al., 2021).

\begin{tabular}{lcccc}
\hline \multicolumn{5}{c}{ Covid-19 death } \\
& YES & NO \\
\hline Moderna vaccine & YES & 0 & 41496 & 41496 \\
& NO & 236 & 3231634 & 3231870 \\
\hline & & 236 & 3273130 & 3273366
\end{tabular}

Statistical analysis.

Causal relationship $\mathrm{k}=\quad-0,0009621672$

$\mathrm{p}$ Value left tailed $(\mathrm{HGD})=0,0492438$

$\mathbf{p}(\mathbf{S I N E})=0,9999279030$

$\tilde{\chi}^{2}\left(\mathrm{SINE}-\mathrm{B}_{\mathrm{t}}\right)=236,0000$

$\tilde{\chi}^{2}\left(\mathrm{SINE}-\underline{\mathrm{A}}_{\mathrm{t}}\right)=\quad 0,0172$

$\mathrm{p}$ Value $(\mathrm{SINE})=0,0000720944$

$\mathbf{p}(\mathbf{I M P})=0,9873231408$

$\tilde{\chi}^{2}\left(\mathrm{IMP}-\mathrm{A}_{\mathrm{t}}\right)=41.496,0000$

$\tilde{\chi}^{2}\left(\mathrm{IMP}-\underline{\mathrm{B}}_{\mathrm{t}}\right)=\quad 526,0769$

$\mathrm{p}$ Value $(\mathrm{IMP})=0,0125968463$

$\mathbf{p}(\mathbf{S I N E} \cap \mathbf{I M P})=0,9872510437$

$\tilde{\chi}^{2}(\mathrm{SINE \cap IMP})_{1}=41.496,0172$

$\tilde{\chi}^{2}(\mathrm{SINE} \cap \mathrm{IMP})_{2}=\quad 762,0769$

$\mathrm{p}$ Value $(\mathrm{SINE \cap IMP})=0,0127$

$\mathbf{p}(\mathbf{E X C L})=1,0000000000$

p $($ EXCL $)$ approx. $=1,0000000000$

$\tilde{\chi}^{2}\left(\mathrm{EXCL}-\mathrm{A}_{\mathrm{t}}\right)=0,0000$

$\tilde{\chi}^{2}\left(\mathrm{EXCL}-\mathrm{B}_{\mathrm{t}}\right)=\quad 0,0000$

$\mathrm{p}$ Value $(\mathrm{EXCL})=0,0000000000$

Relative risk (RR).

$\mathrm{RR}(\mathrm{nc})=\quad 0,0000$

$\mathrm{RR}(\mathrm{sc})=\quad 0,0000$

Additional measures.

$$
\begin{array}{rc}
\mathrm{OR}= & 0,9873 \\
\mathrm{IOR}= & -1,0000
\end{array}
$$

Study design.

$\mathrm{p}(\mathrm{IOU})=0,987251044$

$\mathrm{p}(\mathrm{IOI})=0,012604762$

$\tilde{\chi}^{2}(\mathrm{p}(\mathrm{IOU})=\mathrm{p}(\mathrm{IOI}))=1.636 .211,0340$ 
2.2.4. BionTech

Table 3. BionTech vaccine and Covid-19 death (Study Grange et al., 2021).

\begin{tabular}{lllll}
\hline & \multicolumn{4}{c}{ Covid-19 death } \\
& YES & NO \\
\hline BionTech vaccince & YES & 47 & 1246979 & 1247026 \\
& NO & 189 & 2026151 & 2026340 \\
\hline & & 236 & 3273130 & 3273366
\end{tabular}

Statistical analysis.

Causal relationship $\mathrm{k}=\quad-0,0031789961$

$\mathrm{p}$ Value left tailed (HGD) $=\quad 0,0000000$

$\mathbf{p}($ SINE $)=0,9999422613$

$\tilde{\chi}^{2}\left(\mathrm{SINE}-\mathrm{B}_{\mathrm{t}}\right)=\quad 151,3602$

$\tilde{\chi}^{2}\left(\mathrm{SINE}-\underline{\mathrm{A}}_{\mathrm{t}}\right)=\quad 0,0176$

$\mathrm{p}$ Value $(\mathrm{SINE})=0,0000577371$

$\mathbf{p}(\mathbf{I M P})=0,6190529870$

$\tilde{\chi}^{2}\left(\mathrm{IMP}-\mathrm{A}_{\mathrm{t}}\right)=1.246 .932,0018$

$\tilde{\chi}^{2}\left(\mathrm{IMP}-\underline{\mathrm{B}}_{\mathrm{t}}\right)=475.067,1762$

$\mathrm{p}$ Value $(\mathrm{IMP})=0,3167859098$

$\mathbf{p}(\mathbf{S I N E} \cap \mathbf{I M P})=0,6189952483$

$\tilde{\chi}^{2}(\mathrm{SINE} \cap \mathrm{IMP})_{1}=1.246 .932,0194$

$\tilde{\chi}^{2}(\mathrm{SINE \cap IMP})_{2}=475.218,5364$

$\mathrm{p}$ Value $(\mathrm{SINE \cap IMP})=\quad 0,3168$

$\mathbf{p}(\mathbf{E X C L})=0,9999856417$

p $($ EXCL $)$ approx. $=\quad 0,9999623103$

$\tilde{\chi}^{2}\left(\mathrm{EXCL}-\mathrm{A}_{\mathrm{t}}\right)=0,0018$

$\tilde{\chi}^{2}\left(\mathrm{EXCL}-\mathrm{B}_{\mathrm{t}}\right)=\quad 9,3602$

$\mathrm{p}$ Value $(\mathrm{EXCL})=0,0000143582$

Relative risk (RR).

$$
\begin{array}{ll}
\mathrm{RR}(\mathrm{nc})= & 0,4041 \\
\mathrm{RR}(\mathrm{sc})= & 0,5227
\end{array}
$$

Additional measures.

$$
\begin{array}{rr}
\mathrm{OR}= & 0,6190 \\
\mathrm{IOR}= & -0,4772
\end{array}
$$

\section{Study design.}

$\mathrm{p}(\mathrm{IOU})=0,618966532$

$\mathrm{p}(\mathrm{IOI})=0,380889274$

$\tilde{\chi}^{2}(\mathrm{p}(\mathrm{IOU})=\mathrm{p}(\mathrm{IOI}))=1.636 .211,0340$ 
2.2.5. AstraZeneca

Table 4. AstraZeneca vaccine and Covid-19 death (Study Grange et al. , 2021 ).

\begin{tabular}{lcccc}
\hline & \multicolumn{4}{c}{ Covid-19 death } \\
& & YES & NO & \\
\hline AstraZeneca vaccine & YES & 188 & 2026010 & 2026198 \\
& NO & 48 & 1247120 & 1247168 \\
\hline & & 236 & 3273130 & 3273366
\end{tabular}

\section{Statistical analysis.}

Causal relationship $\mathrm{k}=0,0031055961$ $\mathrm{p}$ Value left tailed $(\mathrm{HGD})=1,0000000$ $\mathbf{p}($ SINE $)=0,9999853362$

$\tilde{\chi}^{2}\left(\mathrm{SINE}-\mathrm{B}_{\mathrm{t}}\right)=\quad 9,7627$

$\tilde{\chi}^{2}\left(\mathrm{SINE}-\underline{\mathrm{A}}_{\mathrm{t}}\right)=\quad 0,0018$

$\mathrm{p}$ Value $(\mathrm{SINE})=0,0000146637$

$\mathbf{p}($ IMP $)=0,3810621849$

$\tilde{\chi}^{2}\left(\mathrm{IMP}-\mathrm{A}_{\mathrm{t}}\right)=2.025 .822,0174$

$\tilde{\chi}^{2}\left(\mathrm{IMP}-\underline{\mathrm{B}}_{\mathrm{t}}\right)=1.254 .064,6171$

$\mathrm{p}$ Value $(\mathrm{IMP})=0,4614838624$

$\mathbf{p}(\mathbf{S I N E} \cap \mathbf{I M P})=0,3810475211$

$\tilde{\chi}^{2}(\mathrm{SINE \cap IMP})_{1}=2.025 .822,0193$

$\tilde{\chi}^{2}(\mathrm{SINE \cap IMP})_{2}=1.254 .074,3798$

$\mathrm{p}$ Value $(\mathrm{SINE} \cap \mathrm{IMP})=\quad 0,4615$

$\mathbf{p}(\mathbf{E X C L})=0,9999425668$

p $($ EXCL) approx. $=0,9999072154$

$\tilde{\chi}^{2}\left(\mathrm{EXCL}-\mathrm{A}_{\mathrm{t}}\right)=0,0174$

$\tilde{\chi}^{2}\left(\mathrm{EXCL}-\mathrm{B}_{\mathrm{t}}\right)=\quad 149,7627$

$\mathrm{p}$ Value $(\mathrm{EXCL})=0,0000574316$

Relative risk ( $R R)$.

$$
\begin{array}{ll}
\mathrm{RR}(\mathrm{nc})= & 2,4108 \\
\mathrm{RR}(\mathrm{sc})= & 1,2870
\end{array}
$$

Additional measures.

$$
\begin{array}{rr}
\text { OR }= & 0,3810 \\
\mathrm{IOR}= & 0,2869
\end{array}
$$

\section{Study design.}

$$
\mathrm{p}(\mathrm{IOU})=0,380932655
$$$$
\mathrm{p}(\mathrm{IOI})=0,618923151
$$

$\tilde{\chi}^{2}(\mathrm{p}(\mathrm{IOU})=\mathrm{p}(\mathrm{IOI}))=1.636 .211,0340$ 
2.2.6. ... and again AstraZeneca Covid-19 vaccine

The study design (see table 4) has been adopted. About 3288252 people in Scotland were not vaccinated at all or at least not by the Covid-19 vaccine of AstraZeneca. The data of able 4 provide evidence that the Covid-19 vaccine of AstraZeneca protects against the Covid-19 death.

Table 5. AstraZeneca vaccine and Covid-19 death (Study Grange et al. , 2021).

\begin{tabular}{lcccc}
\hline & \multicolumn{4}{c}{ Covid-19 death } \\
& & YES & NO \\
\hline AstraZeneca vaccine & YES & 188 & 2026010 & 2026198 \\
& NO & 48662 & 3239590 & 3288252 \\
\hline & & 48850 & 5265600 & 5314450
\end{tabular}

Statistical analysis.

Causal relationship $\mathrm{k}=-0,0748446286$

$\mathrm{p}$ Value left tailed $($ HGD) $=0,0000000$

$\mathbf{p}($ SINE $)=0,9908434551$

$\tilde{\chi}^{2}\left(\operatorname{SINE}-\mathrm{B}_{\mathrm{t}}\right)=48.474,7235$

$\tilde{\chi}^{2}\left(\mathrm{SINE}-\underline{\mathrm{A}}_{\mathrm{t}}\right)=720,1365$

$\mathrm{p}$ Value $(\mathrm{SINE})=0,0091147514$

$\mathbf{p}(\mathbf{I M P})=0,6187733444$

$\tilde{\chi}^{2}\left(\mathrm{IMP}-\mathrm{A}_{\mathrm{t}}\right)=2.025 .822,0174$

$\tilde{\chi}^{2}\left(\mathrm{IMP}-\underline{\mathrm{B}}_{\mathrm{t}}\right)=779.534,4348$

$\mathrm{p}$ Value $(\mathrm{IMP})=0,3169769389$

$\mathbf{p}(\mathbf{S I N E} \cap \mathbf{I M P})=0,6096167995$

$\tilde{\chi}^{2}(\text { SINEกIMP })_{1}=2.026 .542,1539$

$\tilde{\chi}^{2}(\mathrm{SINE} \cap \mathrm{IMP})_{2}=828.009,1584$

$\mathrm{p}$ Value $($ SINEกIMP $)=0,3232$

$\mathbf{p}(\mathbf{E X C L})=0,9999646247$

p $($ EXCL $)$ approx. $=0,9999072154$

$\tilde{\chi}^{2}\left(\mathrm{EXCL}-\mathrm{A}_{\mathrm{t}}\right)=0,0174$

$\tilde{\chi}^{2}\left(\mathrm{EXCL}-\mathrm{B}_{\mathrm{t}}\right)=\quad 0,7235$

$\mathrm{p}$ Value $(\mathrm{EXCL})=0,0000353746$

Relative risk (RR).

$\mathrm{RR}(\mathrm{nc})=0,0063$

$\mathrm{RR}(\mathrm{sc})=\quad 0,0100$

Additional measures.

$\mathrm{OR}=\quad 0,6096$

IOR $=\quad-0,9899$

Study design.

$\mathrm{p}(\mathrm{IOU})=0,609546049$

$\mathrm{p}(\mathrm{IOI})=0,372070111$

$\tilde{\chi}^{2}(\mathrm{p}(\mathrm{IOU})=\mathrm{p}(\mathrm{IOI}))=2.560 .423,0506$ 
The data are presented by table 5 are assuming very conservatively, to the detriment of the AstraZeneca Covid-19 vaccination, that about 1,5 per cent or 48662 of these 3288252 people will die because of a Covid-19 infection. The study design with $\mathrm{p}(\mathrm{IOI})=0,372070111$ is not very convincing but of use to work out the basic relationship between AstraZeneca vaccine and Covid-19 death.

\subsection{Methods}

The nature of definitions is discussed by scientist since ancient times. Many times, several different kinds of definitions are necessary to solve a scientific issue properly. Even if often in play, inappropriate definitions can lead to logical fallacies too.

\subsubsection{The number +0}

Definition 2.1 (The number +0). Let $i$ denote the imaginary number (Bombelli, 1579). The imaginary number $i$ is known to be defined solely by the property that its square is -1. According to today valid rules of algebra, the number +0 is defined as the expression

$$
+0 \equiv+1 \times+0 \equiv+0 \times+1 \equiv+1-1 \equiv+1+i^{2} \equiv+1+e^{i \pi} \equiv \neg(+1)
$$

while ' $=$ 'or $\equiv$ denotes the equals sign (Recorde, 1557) or equality sign (Rolle, 1690) used to indicate equality and '- '(Pacioli, 1494, Widmann, 1489) denotes minus signs used to represent the operations of subtraction and the notions of negative as well and '+ 'denotes the plus (Recorde, 1557) signs used to represent the operations of addition and the notions of positive as well. Negation is denoted by $\neg$.

Remark 2.1. Roger Cotes (1682 - 1716) (Cotes and Halley, 1714) or Leonhard Euler's (1707 - 1783) identity (Euler, 1748) is regarded as one of the most beautiful equations (Wilson, 2018). In this context, it is provisionally presumed, that Euler's identity (Euler, 1748) is logically sound and correct.

\subsubsection{The number +1}

Definition 2.2 (The number +1). According to today valid rules of algebra, the number +1 is defined as the expression

$$
+1 \equiv+1+0 \equiv+1-0 \equiv \neg(+0)
$$

while again '= 'or $\equiv$ may denote the equals sign (Recorde, 1557) or equality sign (Rolle, 1690) used to indicate equality and '- '(Pacioli, 1494, Widmann, 1489) denotes minus signs used to represent the operations of subtraction and the notions of negative as well and ' + 'denotes the plus (Recorde, 1557) signs used to represent the operations of addition and the notions of positive as well.

\subsubsection{Single event distribution}

Let a random variable(Gosset, 1914) X denote something like a function defined on a probability space, which itself maps from the sample space(Neyman and Pearson, 1933) to the real numbers. A single event distribution is more or less a discrete probability distribution of any random variable $\mathrm{X}$ which takes a certain (observer independent) single value $X_{t}$ at a Bernoulli trial(Uspensky, 1937, $p$. 45) (period of time) $t$ with the probability $p\left(X_{t}\right)$. The same random variable $X$ takes a certain single anti value $\underline{X}_{t}$ at a Bernoulli trial (period of time) $t$ with the probability 1-p $\left(X_{t}\right)$. There are conditions in 
nature where a random variable $X$ can take only the values either +0 or +1 . Under these conditions the random variable $\mathrm{X}$ takes the value 1 with probability $\mathrm{p}\left(\mathrm{X}_{\mathrm{t}}=+1\right)$ and the value 0 with probability $q\left(X_{\mathrm{t}}=\right.$ $+0)=1-p\left(X_{\mathrm{t}}=+1\right)$ while the single event distribution passes over into the Bernoulli distribution, named after Swiss mathematician Jacob Bernoulli(Bernoulli, 1713). Less formally, many times, the Bernoulli distribution is represented by a (possibly not biased) coin toss where 1 and 0 would represent 'heads' and 'tails'(or vice versa), respectively. However, the relationship between random variables (Gosset, 1914) can be investigated by many (Gosset, 1908) methods, including the tools of probability theory, too.

\section{Definition 2.3 (Two by two table of single event random variables).}

The two by two or contingency table which has been introduced by Karl Pearson(Pearson, 1904) in 1904 harbours still a large variety of topics and debates. Central to this is the problem to apply the laws of classical logic on data sets, which concerns the justification of inferences which extrapolate from sample data to general facts. Nevertheless, a contingency table is still an appropriate theoretical model too for studying the relationships between random variables, including Bernoulli(Bernoulli, 1713) (i.e. $+0 /+1)$ distributed random variables existing or occurring at the same Bernoulli trial (Uspensky, 1937) (period of time) $\mathrm{t}$.

In this context, let a random variable A at the Bernoulli trial (Uspensky, 1937) (period of time) t, denoted by $A_{t}$, indicate a risk factor, a condition, a cause et cetera and occur or exist with the probability $\mathrm{p}\left(\mathrm{A}_{\mathrm{t}}\right)$ at the Bernoulli trial (Uspensky, 1937) (period of time) t. Let $\mathrm{E}\left(\mathrm{A}_{t}\right)$ denote the expectation value of $A_{t}$. In general it is

$$
p\left(A_{\mathrm{t}}\right) \equiv p\left(a_{\mathrm{t}}\right)+p\left(b_{\mathrm{t}}\right)
$$

The expectation value $E\left(A_{t}\right)$ follows as

$$
\begin{aligned}
E\left(A_{\mathrm{t}}\right) & \equiv A_{\mathrm{t}} \times p\left(A_{\mathrm{t}}\right) \\
& \equiv A_{\mathrm{t}} \times\left(p\left(a_{\mathrm{t}}\right)+p\left(b_{\mathrm{t}}\right)\right) \\
& \equiv\left(A_{\mathrm{t}} \times p\left(a_{\mathrm{t}}\right)\right)+\left(A_{\mathrm{t}} \times p\left(b_{\mathrm{t}}\right)\right) \\
& \equiv E\left(a_{\mathrm{t}}\right)+E\left(b_{\mathrm{t}}\right)
\end{aligned}
$$

Under conditions of $+0 /+1$ distributed Bernoulli random variables it is

$$
\begin{aligned}
E\left(A_{\mathrm{t}}\right) & \equiv A_{\mathrm{t}} \times p\left(A_{\mathrm{t}}\right) \\
& \equiv(+0+1) \times p\left(A_{\mathrm{t}}\right) \\
& \equiv p\left(A_{\mathrm{t}}\right) \\
& \equiv p\left(a_{\mathrm{t}}\right)+p\left(b_{\mathrm{t}}\right)
\end{aligned}
$$

Furthermore, it is

$$
p\left(\underline{A}_{\mathrm{t}}\right) \equiv p\left(c_{\mathrm{t}}\right)+p\left(d_{\mathrm{t}}\right) \equiv\left(1-p\left(A_{\mathrm{t}}\right)\right)
$$

The expectation value $\mathrm{E}\left(\underline{\mathrm{A}}_{\mathrm{t}}\right)$ is given as

$$
\begin{aligned}
E\left(\underline{A}_{\mathrm{t}}\right) & \equiv A_{\mathrm{t}} \times\left(1-p\left(A_{\mathrm{t}}\right)\right) \\
& \equiv A_{\mathrm{t}} \times\left(p\left(c_{\mathrm{t}}\right)+p\left(d_{\mathrm{t}}\right)\right) \\
& \equiv\left(A_{\mathrm{t}} \times p\left(c_{\mathrm{t}}\right)\right)+\left(A_{\mathrm{t}} \times p\left(d_{\mathrm{t}}\right)\right) \\
& \equiv E\left(c_{\mathrm{t}}\right)+E\left(d_{\mathrm{t}}\right)
\end{aligned}
$$


Under conditions of $+0 /+1$ distributed Bernoulli random variables we obtain

$$
\begin{aligned}
E\left(\underline{A}_{\mathrm{t}}\right) & \equiv A_{\mathrm{t}} \times\left(1-p\left(A_{\mathrm{t}}\right)\right) \\
& \equiv(+0+1) \times\left(1-p\left(A_{\mathrm{t}}\right)\right) \\
& \equiv\left(1-p\left(A_{\mathrm{t}}\right)\right) \\
& \equiv p\left(c_{\mathrm{t}}\right)+p\left(d_{\mathrm{t}}\right)
\end{aligned}
$$

Let a random variable $\mathrm{B}$ at the Bernoulli trial (Uspensky, 1937) (period of time) t, denoted by $\mathrm{B}_{\mathrm{t}}$, indicate an outcome, a conditioned, an effect et cetera and occur or exist with the probability $\mathrm{p}\left(\mathrm{B}_{\mathrm{t}}\right)$ at the Bernoulli trial (Uspensky, 1937) (period of time) t. Let $\mathrm{E}\left(\mathrm{B}_{\mathrm{t}}\right)$ denote the expectation value of $\mathrm{B}_{\mathrm{t}}$. In general it is

$$
p\left(B_{\mathrm{t}}\right) \equiv p\left(a_{\mathrm{t}}\right)+p\left(c_{\mathrm{t}}\right)
$$

The expectation value $\mathrm{E}\left(\mathrm{B}_{\mathrm{t}}\right)$ is given by the equation

$$
\begin{aligned}
E\left(B_{\mathrm{t}}\right) & \equiv B_{\mathrm{t}} \times p\left(B_{\mathrm{t}}\right) \\
& \equiv B_{\mathrm{t}} \times\left(p\left(a_{\mathrm{t}}\right)+p\left(c_{\mathrm{t}}\right)\right) \\
& \equiv\left(B_{\mathrm{t}} \times p\left(a_{\mathrm{t}}\right)\right)+\left(B_{\mathrm{t}} \times p\left(c_{\mathrm{t}}\right)\right) \\
& \equiv E\left(a_{\mathrm{t}}\right)+E\left(c_{\mathrm{t}}\right)
\end{aligned}
$$

Under conditions of $+0 /+1$ distributed Bernoulli random variables it is

$$
\begin{aligned}
E\left(B_{\mathrm{t}}\right) & \equiv B_{\mathrm{t}} \times p\left(B_{\mathrm{t}}\right) \\
& \equiv(+0+1) \times p\left(B_{\mathrm{t}}\right) \\
& \equiv p\left(B_{\mathrm{t}}\right) \\
& \equiv p\left(a_{\mathrm{t}}\right)+p\left(c_{\mathrm{t}}\right)
\end{aligned}
$$

Furthermore, it is

$$
p\left(\underline{B}_{\mathrm{t}}\right) \equiv p\left(b_{\mathrm{t}}\right)+p\left(d_{\mathrm{t}}\right) \equiv\left(1-p\left(B_{\mathrm{t}}\right)\right)
$$

The expectation value $\mathrm{E}\left(\underline{B}_{t}\right)$ is given by the equation

$$
\begin{aligned}
E\left(\underline{B}_{\mathrm{t}}\right) & \equiv B_{\mathrm{t}} \times\left(1-p\left(B_{\mathrm{t}}\right)\right) \\
& \equiv B_{\mathrm{t}} \times\left(p\left(b_{\mathrm{t}}\right)+p\left(d_{\mathrm{t}}\right)\right) \\
& \equiv\left(B_{\mathrm{t}} \times p\left(b_{\mathrm{t}}\right)\right)+\left(B_{\mathrm{t}} \times p\left(d_{\mathrm{t}}\right)\right) \\
& \equiv E\left(b_{\mathrm{t}}\right)+E\left(d_{\mathrm{t}}\right)
\end{aligned}
$$

Under conditions of $+0 /+1$ distributed Bernoulli random variables it is

$$
\begin{aligned}
E\left(\underline{B}_{\mathrm{t}}\right) & \equiv B_{\mathrm{t}} \times\left(1-p\left(B_{\mathrm{t}}\right)\right) \\
& \equiv(+0+1) \times\left(1-p\left(B_{\mathrm{t}}\right)\right) \\
& \equiv\left(1-p\left(B_{\mathrm{t}}\right)\right) \\
& \equiv p\left(b_{\mathrm{t}}\right)+p\left(d_{\mathrm{t}}\right)
\end{aligned}
$$


Let $p\left(a_{t}\right)=p\left(A_{t} \wedge B_{t}\right)$ denote the joint probability distribution of $A_{t}$ and $B_{t}$ at the same Bernoulli trial (period of time) t. In general, it is

$$
\begin{aligned}
E\left(a_{\mathrm{t}}\right) & \equiv E\left(A_{\mathrm{t}} \wedge B_{\mathrm{t}}\right) \\
& \equiv\left(A_{\mathrm{t}} \times B_{\mathrm{t}}\right) \times p\left(A_{\mathrm{t}} \wedge B_{\mathrm{t}}\right) \\
& \equiv\left(A_{\mathrm{t}} \times B_{\mathrm{t}}\right) \times p\left(a_{\mathrm{t}}\right)
\end{aligned}
$$

Under conditions of $+0 /+1$ distributed Bernoulli random variables, it is

$$
\begin{aligned}
E\left(a_{\mathrm{t}}\right) & \equiv E\left(A_{\mathrm{t}} \wedge B_{\mathrm{t}}\right) \\
& \equiv\left(A_{\mathrm{t}} \times B_{\mathrm{t}}\right) \times p\left(A_{\mathrm{t}} \wedge B_{\mathrm{t}}\right) \\
& \equiv((+0+1) \times(+0+1)) \times p\left(A_{\mathrm{t}} \wedge B_{\mathrm{t}}\right) \\
& \equiv p\left(A_{\mathrm{t}} \wedge B_{\mathrm{t}}\right) \\
& \equiv p\left(a_{\mathrm{t}}\right)
\end{aligned}
$$

Let $\mathrm{p}\left(\mathrm{b}_{t}\right)=\mathrm{p}\left(\mathrm{A}_{\mathrm{t}} \wedge \neg \mathrm{B}_{\mathrm{t}}\right)$ denote the joint probability distribution of $\mathrm{A}_{t}$ and not $\mathrm{B}_{\mathrm{t}}$ at the same Bernoulli trial (period of time) t. In general, it is

$$
\begin{aligned}
E\left(b_{\mathrm{t}}\right) & \equiv E\left(A_{\mathrm{t}} \wedge \neg B_{\mathrm{t}}\right) \\
& \equiv\left(A_{\mathrm{t}} \times \neg B_{\mathrm{t}}\right) \times p\left(A_{\mathrm{t}} \wedge \neg B_{\mathrm{t}}\right) \\
& \equiv\left(A_{\mathrm{t}} \times \neg B_{\mathrm{t}}\right) \times p\left(b_{\mathrm{t}}\right)
\end{aligned}
$$

Under conditions of $+0 /+1$ distributed Bernoulli random variables, it is

$$
\begin{aligned}
E\left(b_{\mathrm{t}}\right) & \equiv E\left(A_{\mathrm{t}} \wedge \neg B_{\mathrm{t}}\right) \\
& \equiv\left(A_{\mathrm{t}} \times \neg B_{\mathrm{t}}\right) \times p\left(A_{\mathrm{t}} \wedge \neg B_{\mathrm{t}}\right) \\
& \equiv((+0+1) \times(+0+1)) \times p\left(A_{\mathrm{t}} \wedge \neg B_{\mathrm{t}}\right) \\
& \equiv p\left(A_{\mathrm{t}} \wedge \neg B_{\mathrm{t}}\right) \\
& \equiv p\left(b_{\mathrm{t}}\right)
\end{aligned}
$$

Let $\mathrm{p}\left(\mathrm{c}_{\mathrm{t}}\right)=\mathrm{p}\left(\neg \mathrm{A}_{\mathrm{t}} \wedge \mathrm{B}_{t}\right)$ denote the joint probability distribution of not $\mathrm{A}_{t}$ and $\mathrm{B}_{t}$ at the same Bernoulli trial (period of time) $t$. In general, it is

$$
\begin{aligned}
E\left(c_{\mathrm{t}}\right) & \equiv E\left(\neg A_{\mathrm{t}} \wedge B_{\mathrm{t}}\right) \\
& \equiv\left(\neg A_{\mathrm{t}} \wedge B_{\mathrm{t}}\right) \times p\left(\neg A_{\mathrm{t}} \wedge B_{\mathrm{t}}\right) \\
& \equiv\left(\neg A_{\mathrm{t}} \wedge B_{\mathrm{t}}\right) \times p\left(c_{\mathrm{t}}\right)
\end{aligned}
$$

Under conditions of $+0 /+1$ distributed Bernoulli random variables, it is

$$
\begin{aligned}
E\left(c_{\mathrm{t}}\right) & \equiv E\left(\neg A_{\mathrm{t}} \wedge B_{\mathrm{t}}\right) \\
& \equiv\left(\neg A_{\mathrm{t}} \times B_{\mathrm{t}}\right) \times p\left(\neg A_{\mathrm{t}} \wedge B_{\mathrm{t}}\right) \\
& \equiv((+0+1) \times(+0+1)) \times p\left(\neg A_{\mathrm{t}} \wedge B_{\mathrm{t}}\right) \\
& \equiv p\left(\neg A_{\mathrm{t}} \wedge B_{\mathrm{t}}\right) \\
& \equiv p\left(c_{\mathrm{t}}\right)
\end{aligned}
$$


Let $\mathrm{p}\left(\mathrm{d}_{\mathrm{t}}\right)=\mathrm{p}\left(\neg \mathrm{A}_{\mathrm{t}} \wedge \neg \mathrm{B}_{\mathrm{t}}\right)$ denote the joint probability distribution of not $\mathrm{A}_{\mathrm{t}}$ and not $\mathrm{B}_{\mathrm{t}}$ at the same Bernoulli trial (period of time) t. In general, it is

$$
\begin{aligned}
E\left(d_{\mathrm{t}}\right) & \equiv E\left(\neg A_{\mathrm{t}} \times \neg B_{\mathrm{t}}\right) \\
& \equiv\left(\neg A_{\mathrm{t}} \times \neg B_{\mathrm{t}}\right) \times p\left(\neg A_{\mathrm{t}} \wedge \neg B_{\mathrm{t}}\right) \\
& \equiv\left(\neg A_{\mathrm{t}} \times \neg B_{\mathrm{t}}\right) \times p\left(d_{\mathrm{t}}\right)
\end{aligned}
$$

Under conditions of $+0 /+1$ distributed Bernoulli random variables, it is

$$
\begin{aligned}
E\left(d_{\mathrm{t}}\right) & \equiv E\left(\neg A_{\mathrm{t}} \wedge \neg B_{\mathrm{t}}\right) \\
& \equiv\left(\neg A_{\mathrm{t}} \times \neg B_{\mathrm{t}}\right) \times p\left(\neg A_{\mathrm{t}} \wedge \neg B_{\mathrm{t}}\right) \\
& \equiv((+0+1) \times(+0+1)) \times p\left(\neg A_{\mathrm{t}} \wedge \neg B_{\mathrm{t}}\right) \\
& \equiv p\left(\neg A_{\mathrm{t}} \wedge \neg B_{\mathrm{t}}\right) \\
& \equiv p\left(d_{\mathrm{t}}\right)
\end{aligned}
$$

In general, it is

$$
p\left(a_{\mathrm{t}}\right)+p\left(b_{\mathrm{t}}\right)+p\left(c_{\mathrm{t}}\right)+p\left(d_{\mathrm{t}}\right) \equiv+1
$$

Table 6 provide us with an overview of the definitions above.

Table 6. The two by two table of Bernoulli random variables

\begin{tabular}{ccccc} 
& & \multicolumn{3}{c}{ Conditioned $\mathrm{B}_{\mathrm{t}}$} \\
& & TRUE & FALSE \\
\hline Condition & TRUE & $\mathrm{p}\left(\mathrm{a}_{\mathrm{t}}\right)$ & $\mathrm{p}\left(\mathrm{b}_{\mathrm{t}}\right)$ & $\mathrm{p}\left(\mathrm{A}_{\mathrm{t}}\right)$ \\
$\mathrm{A}_{\mathrm{t}}$ & FALSE & $\mathrm{p}\left(\mathrm{c}_{\mathrm{t}}\right)$ & $\mathrm{p}\left(\mathrm{d}_{\mathrm{t}}\right)$ & $\mathrm{p}\left(\underline{\mathrm{A}}_{\mathrm{t}}\right)$ \\
\hline & & $\mathrm{p}\left(\mathrm{B}_{\mathrm{t}}\right)$ & $\mathrm{p}\left(\underline{\mathrm{B}}_{\mathrm{t}}\right)$ & +1
\end{tabular}

\subsubsection{Binomial random variables}

\section{Definition 2.4 (Two by two table of Binomial random variables).}

Let $\mathrm{a}, \mathrm{b}, \mathrm{c}, \mathrm{d}, \mathrm{A}, \underline{\mathrm{A}}, \mathrm{B}$, and $\underline{\mathrm{B}}$ denote expectation values. Under conditions where the probability of an event, an outcome, a success et cetera is constant from Bernoulli trial to Bernoulli trial $t$, it is

$$
\begin{aligned}
A & =N \times E\left(A_{\mathrm{t}}\right) \\
& \equiv N \times\left(A_{\mathrm{t}} \times p\left(A_{\mathrm{t}}\right)\right) \\
& \equiv N \times\left(p\left(A_{\mathrm{t}}\right)+p\left(B_{\mathrm{t}}\right)\right) \\
& \equiv N \times p\left(A_{\mathrm{t}}\right)
\end{aligned}
$$

and 


$$
\begin{aligned}
B & =N \times E\left(B_{\mathrm{t}}\right) \\
& \equiv N \times\left(B_{\mathrm{t}} \times p\left(B_{\mathrm{t}}\right)\right) \\
& \equiv N \times\left(p\left(A_{\mathrm{t}}\right)+p\left(c_{\mathrm{t}}\right)\right) \\
& \equiv N \times p\left(B_{\mathrm{t}}\right)
\end{aligned}
$$

where $\mathrm{N}$ might denote the population or even the sample size. Furthermore, it is

$$
a \equiv N \times\left(E\left(A_{\mathrm{t}}\right)\right) \equiv N \times\left(p\left(A_{\mathrm{t}}\right)\right)
$$

and

$$
b \equiv N \times\left(E\left(B_{\mathrm{t}}\right)\right) \equiv N \times\left(p\left(B_{\mathrm{t}}\right)\right)
$$

and

$$
c \equiv N \times\left(E\left(c_{\mathrm{t}}\right)\right) \equiv N \times\left(p\left(c_{\mathrm{t}}\right)\right)
$$

and

$$
d \equiv N \times\left(E\left(d_{\mathrm{t}}\right)\right) \equiv N \times\left(p\left(d_{\mathrm{t}}\right)\right)
$$

and

$$
a+b+c+d \equiv A+\underline{A} \equiv B+\underline{B} \equiv N
$$

Table 7 provide us again an overview of a two by two table of Binomial random variables.

Table 7. The two by two table of Binomial random variables

\begin{tabular}{ccccc} 
& & \multicolumn{3}{c}{ Conditioned B } \\
& & TRUE & FALSE \\
\hline Condition & TRUE & $\mathrm{a}$ & $\mathrm{b}$ & $\mathrm{A}$ \\
$\mathrm{A}_{\mathrm{t}}$ & FALSE & $\mathrm{c}$ & $\mathrm{d}$ & $\underline{\mathrm{A}}$ \\
\hline & & $\mathrm{B}$ & $\underline{\mathrm{B}}$ & $\underline{\mathrm{N}}$
\end{tabular}

\subsubsection{Independence}

\section{Definition 2.5 (Independence).}

In general, an event $A_{t}$ at the Bernoulli trial $t$ need not but can be independent of the existence or of the occurrence of another event $\mathrm{B}_{\mathrm{t}}$ at the same Bernoulli trial $\mathrm{t}$. Mathematically, independence (Kolmogoroff, 1933, Moivre, 1718) in terms of probability theory is defined at the same (period of) time $\mathrm{t}$ (i.e. Bernoulli trial $\mathrm{t}$ ) as

$$
\begin{aligned}
p\left(A_{\mathrm{t}} \wedge B_{\mathrm{t}}\right) & \equiv p\left(A_{\mathrm{t}}\right) \times p\left(B_{\mathrm{t}}\right) \\
& \equiv \frac{\sum_{t=1}^{N}\left(A_{\mathrm{t}} \wedge B_{\mathrm{t}}\right)}{N} \equiv \frac{N \times\left(p\left(a_{\mathrm{t}}\right)\right)}{N} \equiv 1-p\left(A_{\mathrm{t}} \mid B_{\mathrm{t}}\right) \equiv 1-p\left(A_{\mathrm{t}} \uparrow B_{\mathrm{t}}\right)
\end{aligned}
$$


2.3.6. Dependence

Definition 2.6 (Dependence).

The dependence of events (Barukčić, 1989, p. 57-61) is defined as

$$
p(\underbrace{A_{\mathrm{t}} \wedge B_{\mathrm{t}} \wedge C_{\mathrm{t}} \wedge \ldots}_{n}) \equiv \sqrt[n]{\underbrace{p\left(A_{\mathrm{t}}\right) \times p\left(B_{\mathrm{t}}\right) \times p\left(C_{\mathrm{t}}\right) \times \ldots}_{n}}
$$

2.3.7. Exclusion relationship

\section{Definition 2.7 (Exclusion relationship [EXCL]).}

Mathematically, the exclusion (EXCL) relationship, denoted by $\mathrm{p}\left(\mathrm{A}_{t} \mid \mathrm{B}_{t}\right)$ in terms of statistics and probability theory, is defined(Barukčić, 1989, p. 68-70) as

$$
\begin{aligned}
p\left(A_{\mathrm{t}} \mid B_{\mathrm{t}}\right) & \equiv p\left(A_{\mathrm{t}} \uparrow B_{\mathrm{t}}\right) \\
& \equiv p\left(b_{\mathrm{t}}\right)+p\left(c_{\mathrm{t}}\right)+p\left(d_{\mathrm{t}}\right) \\
& \equiv \frac{N \times\left(p\left(b_{\mathrm{t}}\right)+p\left(c_{\mathrm{t}}\right)+p\left(d_{\mathrm{t}}\right)\right)}{N} \\
& \equiv \frac{\sum_{t=1}^{N}\left(\underline{A}_{\mathrm{t}} \vee \underline{B} \mathrm{t}\right)}{N} \equiv \frac{b+c+d}{N} \\
& \equiv \frac{b+\underline{A}}{N} \\
& \equiv \frac{c+\underline{B}}{N} \\
& \equiv+1
\end{aligned}
$$

Based on the 1913 Henry Maurice Sheffer (1882-1964) relationship, the Sheffer stroke(Nicod, 1917, Sheffer, 1913) usually denoted by $\uparrow$, it is $p\left(A_{\mathrm{t}} \wedge B_{\mathrm{t}}\right) \equiv 1-p\left(A_{\mathrm{t}} \mid B_{\mathrm{t}}\right)$ (see table 8 ).

Table 8. $A_{t}$ excludes $B_{t}$ and vice versa.

\begin{tabular}{ccccc} 
& \multicolumn{4}{c}{ Conditioned (COVID-19) $\mathrm{B}_{\mathrm{t}}$} \\
& & TRUE & FALSE & \\
\hline Condition (Vaccine) & TRUE & $\mathbf{+ 0}$ & $\mathrm{p}\left(\mathrm{b}_{\mathrm{t}}\right)$ & $\mathrm{p}\left(\mathrm{A}_{\mathrm{t}}\right)$ \\
$\mathrm{A}_{\mathrm{t}}$ & FALSE & $\mathrm{p}\left(\mathrm{c}_{\mathrm{t}}\right)$ & $\mathrm{p}\left(\mathrm{d}_{\mathrm{t}}\right)$ & $\mathrm{p}\left(\underline{\mathrm{A}}_{\mathrm{t}}\right)$ \\
\hline & & $\mathrm{p}\left(\mathrm{B}_{\mathrm{t}}\right)$ & $\mathrm{p}\left(\underline{\mathrm{B}}_{\mathrm{t}}\right)$ & +1
\end{tabular}

Remark 2.2. Pfizer Inc. and BioNTech SE announced on Monday, November 09, 2020 - 06:45am results from a Phase 3 COVID-19 vaccine trial with 43.538 participants which provides evidence that their vaccine (BNT162b2) is preventing COVID-19 in participants without evidence of prior SARSCoV-2 infection. In toto, 170 confirmed cases of COVID-19 were evaluated, with 8 in the vaccine 
group versus 162 in the placebo group. The exclusion relationship can be calculated as follows.

$$
\begin{aligned}
p(\text { Vaccine }: \text { BNT } 162 b 2 \mid \text { COVID }-19(\text { infection })) & \equiv p\left(b_{t}\right)+p\left(c_{t}\right)+p\left(d_{t}\right) \\
& \equiv 1-p\left(a_{t}\right) \\
& \equiv 1-\left(\frac{8}{43538}\right) \\
& \equiv+0,99981625
\end{aligned}
$$

with a P Value $=0,000184$

2.3.8. The goodness of fit test of an exclusion relationship

\section{Definition 2.8 (The $\tilde{\chi}^{2}$ goodness of fit test of an exclusion relationship).}

Under some well known circumstances, testing hypothesis about an exclusion relationship $\mathrm{p}\left(\mathrm{A}_{\mathrm{t}} \mid\right.$ $\mathrm{B}_{\mathrm{t}}$ ) is possible by the chi-square distribution (also chi-squared or $\tilde{\chi}^{2}$-distribution) too. The $\tilde{\chi}^{2}$ goodness of fit test of an exclusion relationship with degree of freedom (d. f.) of d. f. $=1$ is calculated as

$$
\begin{aligned}
\tilde{\chi}_{\text {Calculated }}^{2}\left(\left(A_{\mathrm{t}} \mid B_{\mathrm{t}}\right) \mid A\right) \equiv & \frac{(b-(a+b))^{2}}{A}+ \\
& \frac{((c+d)-\underline{A})^{2}}{\underline{A}} \\
& \equiv \frac{a^{2}}{A}+0 \\
& \equiv \frac{a^{2}}{A}
\end{aligned}
$$

or equally as

$$
\begin{aligned}
\tilde{\chi}^{2} \text { Calculated }\left(\left(A_{\mathrm{t}} \mid B_{\mathrm{t}}\right) \mid B\right) \equiv & \frac{(c-(a+c))^{2}}{B}+ \\
& \frac{((b+d)-\underline{B})^{2}}{\underline{B}} \\
& \equiv \frac{a^{2}}{B}+0 \\
& \equiv \frac{a^{2}}{B}
\end{aligned}
$$

and can be compared with a theoretical chi-square value at a certain level of significance $\alpha$. The $\tilde{\chi}^{2}$-distribution equals zero when the observed values are equal to the expected/theoretical values of an exclusion relationship/distribution $\mathrm{p}\left(\mathrm{A}_{\mathrm{t}} \mid \mathrm{B}_{\mathrm{t}}\right)$, in which case the null hypothesis has to be accepted. Yate's (Yates, 1934) continuity correction was not used under these circumstances. 
2.3.9. The left-tailed $\mathrm{p}$ Value of an exclusion relationship

\section{Definition 2.9 (The left-tailed p Value of an exclusion relationship).}

It is known that as a sample size, $\mathrm{N}$, increases, a sampling distribution of a special test statistic approaches the normal distribution (central limit theorem). Under these circumstances, the left-tailed (lt) p Value (Barukčić, 2019c) of an exclusion relationship can be calculated as follows.

$$
\begin{aligned}
\text { pValue }_{\mathrm{lt}}\left(A_{\mathrm{t}} \mid B_{\mathrm{t}}\right) & \equiv 1-e^{-\left(1-p\left(A_{\mathrm{t}} \mid B_{\mathrm{t}}\right)\right)} \\
& \equiv 1-e^{-(a / N)}
\end{aligned}
$$

A low p-value may provide some evidence of statistical significance.

\subsubsection{Neither nor conditions}

\section{Definition 2.10 (Neither $A_{t}$ nor $B_{t}$ conditions $[N O R]$ ).}

Mathematically, a neither $\mathrm{A}_{\mathrm{t}}$ nor $\mathrm{B}_{\mathrm{t}}$ condition (or rejection according to the French philosopher and logician Jean George Pierre Nicod (1893-1924), i.e. Jean Nicod's statement (Nicod, 1924)) relationship (NOR), denoted by $\mathrm{p}\left(\mathrm{A}_{\mathrm{t}} \downarrow \mathrm{B}_{\mathrm{t}}\right)$ in terms of statistics and probability theory, is defined (Barukčić, 1989, p. 68-70) as

$$
\begin{aligned}
p\left(A_{\mathrm{t}} \downarrow B_{\mathrm{t}}\right) & \equiv p\left(d_{\mathrm{t}}\right) \\
& \equiv \frac{N-\sum_{t=1}^{N}\left(A_{\mathrm{t}} \vee B_{\mathrm{t}}\right)}{N} \equiv \frac{\sum_{t=1}^{N}\left(\underline{A}_{\mathrm{t}} \wedge \underline{B}_{\mathrm{t}}\right)}{N} \equiv \frac{N \times\left(p\left(d_{\mathrm{t}}\right)\right)}{N} \\
& \equiv \frac{d}{N} \\
& \equiv+1
\end{aligned}
$$

2.3.11. The Chi square goodness of fit test of a neither nor condition relationship

\section{Definition 2.11 (The $\tilde{\chi}^{2}$ goodness of fit test of a neither $\mathbf{A}_{t}$ nor $\mathbf{B}_{\mathbf{t}}$ condition relationship).}

A neither $A_{t}$ nor $B_{t}$ condition relationship $p\left(A_{t} \downarrow B_{t}\right)$ can be tested by the chi-square distribution (also chi-squared or $\tilde{\chi}^{2}$-distribution). The $\tilde{\chi}^{2}$ goodness of fit test of a neither $A_{t}$ nor $B_{t}$ condition relationship with degree of freedom (d. f.) of d. f. $=1$ may be calculated as

$$
\begin{aligned}
\tilde{\chi}^{2} \text { Calculated }\left(\left(A_{\mathrm{t}} \downarrow B_{\mathrm{t}}\right) \mid A\right) \equiv & \frac{(d-(c+d))^{2}}{\underline{A}}+ \\
& \frac{((a+b)-A)^{2}}{A} \\
& \equiv \frac{c^{2}}{\underline{A}}+0
\end{aligned}
$$


or equally as

$$
\begin{aligned}
\tilde{\chi}_{\text {Calculated }}^{2}\left(\left(A_{\mathrm{t}} \downarrow B_{\mathrm{t}}\right) \mid B\right) \equiv & \frac{(d-(b+d))^{2}}{\underline{B}}+ \\
& \frac{((a+c)-B)^{2}}{B} \\
& \equiv \frac{b^{2}}{\underline{B}}+0
\end{aligned}
$$

Yate's (Yates, 1934) continuity correction has not been used in this context.

2.3.12. The left-tailed $\mathrm{p}$ Value of a neither nor B condition relationship

Definition 2.12 (The left-tailed $p$ Value of a neither $A_{t}$ nor $B_{t}$ condition relationship).

The left-tailed (lt) p Value (Barukčić, 2019c) of a neither $A_{t}$ nor $B_{t}$ condition relationship can be calculated as follows.

$$
\begin{aligned}
\text { pValue }_{\mathrm{lt}}\left(A_{\mathrm{t}} \downarrow B_{\mathrm{t}}\right) & \equiv 1-e^{-\left(1-p\left(A_{\mathrm{t}} \downarrow B_{\mathrm{t}}\right)\right)} \\
& \equiv 1-e^{-p\left(A_{\mathrm{t}} \vee B_{\mathrm{t}}\right)} \\
& \equiv 1-e^{-((a+b+c) / N)}
\end{aligned}
$$

where $\vee$ may denote disjunction or logical inclusive or. In this context, a low p-value indicates again a statistical significance. In general, it is $p\left(A_{\mathrm{t}} \vee B_{\mathrm{t}}\right) \equiv 1-p\left(A_{\mathrm{t}} \downarrow B_{\mathrm{t}}\right)$ (see table 9).

Table 9. Neither $A_{t}$ nor $B_{t}$ relationship.

\begin{tabular}{llccc}
\hline & \multicolumn{4}{c}{ Conditioned $\mathrm{B}_{\mathrm{t}}$} \\
& & YES & NO \\
\hline Condition $\mathrm{A}_{\mathrm{t}}$ & YES & 0 & 0 & 0 \\
& NO & 0 & 1 & 1 \\
\hline & & 0 & 1 & 1
\end{tabular}

2.3.13. Necessary condition

Definition 2.13 (Necessary condition [Conditio sine qua non]).

Mathematically, the necessary condition (SINE) relationship, denoted by $\mathrm{p}\left(\mathrm{A}_{t} \leftarrow \mathrm{B}_{t}\right.$ ) in terms of 
statistics and probability theory, is defined (Barukčić, 1989, p. 15-28) as

$$
\begin{aligned}
p\left(A_{\mathrm{t}} \leftarrow B_{\mathrm{t}}\right) \equiv p\left(A_{\mathrm{t}} \vee \underline{B} \mathrm{t}\right) & \equiv \frac{\sum_{t=1}^{N}\left(A_{\mathrm{t}} \vee \underline{B}_{\mathrm{t}}\right)}{N} \equiv \frac{\left(A_{\mathrm{t}} \vee \underline{B}_{\mathrm{t}}\right) \times p\left(A_{\mathrm{t}} \vee \underline{B}_{\mathrm{t}}\right)}{\left(A_{\mathrm{t}} \vee \underline{B} \underline{\mathrm{t}}\right)} \\
& \equiv p\left(a_{\mathrm{t}}\right)+p\left(b_{\mathrm{t}}\right)+p\left(d_{\mathrm{t}}\right) \\
& \equiv \frac{N \times\left(p\left(a_{\mathrm{t}}\right)+p\left(b_{\mathrm{t}}\right)+p\left(d_{\mathrm{t}}\right)\right)}{N} \equiv \frac{E\left(A_{\mathrm{t}} \leftarrow B_{\mathrm{t}}\right)}{N} \\
& \equiv \frac{a+b+d}{N} \equiv \frac{E\left(A_{\mathrm{t}} \vee \underline{B}_{\mathrm{t}}\right)}{N} \\
& \equiv \frac{A+d}{N} \equiv \frac{E\left(A_{\mathrm{t}} \leftarrow B_{\mathrm{t}}\right)}{N} \\
& \equiv \frac{a+\underline{B}}{N} \equiv \frac{E\left(A_{\mathrm{t}} \vee \underline{B}_{\mathrm{t}}\right)}{N} \\
& \equiv+1
\end{aligned}
$$

where $E\left(A_{\mathrm{t}} \leftarrow B_{\mathrm{t}}\right) \equiv E\left(A_{\mathrm{t}} \vee \underline{B}_{\mathrm{t}}\right)$ indicates the expectation value of the necessary condition. In general, it is $p\left(A_{\mathrm{t}}<B_{\mathrm{t}}\right) \equiv 1-p\left(A_{\mathrm{t}} \leftarrow B_{\mathrm{t}}\right)$ (see Table 10).

Table 10. Necessary condition.

\begin{tabular}{ccccc} 
& & \multicolumn{3}{c}{ Conditioned $\mathrm{B}_{\mathrm{t}}$} \\
& & TRUE & FALSE \\
\hline Condition & TRUE & $\mathrm{p}\left(\mathrm{a}_{\mathrm{t}}\right)$ & $\mathrm{p}\left(\mathrm{b}_{\mathrm{t}}\right)$ & $\mathrm{p}\left(\mathrm{A}_{\mathrm{t}}\right)$ \\
$\mathrm{A}_{\mathrm{t}}$ & FALSE & $\mathbf{+ 0}$ & $\mathrm{p}\left(\mathrm{d}_{\mathrm{t}}\right)$ & $\mathrm{p}\left(\underline{\mathrm{A}}_{\mathrm{t}}\right)$ \\
\hline & & $\mathrm{p}\left(\mathrm{B}_{\mathrm{t}}\right)$ & $\mathrm{p}\left(\underline{\mathrm{B}}_{\mathrm{t}}\right)$ & +1
\end{tabular}

Remark 2.3. A necessary condition $A_{t}$ is characterized itself by the property that another event $B_{t}$ will not occur if $A_{t}$ is not given, if $A_{t}$ did not occur (Barukčić, 1989, 1997, 2005, 2016, 2017a,b, 2020a,b,c,d, Barukčić and Ufuoma, 2020). Example. A human being cannot live without water. A human being cannot live without gaseous oxygen et cetera. Water itself is a necessary condition of human life. However, gaseous oxygen is a necessary condition of human life too. Thus far, even if water is given and even if water is a necessary condition of human life, without gaseous oxygen there will be no human life. In general, if a conditioned or an outcome $B_{t}$ depends on the necessary condition $A_{t}$ and equally on numerous other necessary conditions, an event $B_{t}$ will not occur if $A_{t}$ itself is not given independently of the occurrence of other necessary conditions.

2.3.14. The Chi-square goodness of fit test of a necessary condition relationship

\section{Definition 2.14 (The $\tilde{\chi}^{2}$ goodness of fit test of a necessary condition relationship).}

Under some well known circumstances, hypothesis about the conditio sine qua non relationship $p\left(A_{t}\right.$ $\leftarrow \mathrm{B}_{\mathrm{t}}$ ) can be tested by the chi-square distribution (also chi-squared or $\chi^{2}$-distribution), first described by the German statistician Friedrich Robert Helmert (Helmert, 1876) and later rediscovered by Karl 
Pearson (Pearson, 1900) in the context of a goodness of fit test. The $\tilde{\chi}^{2}$ goodness of fit test of a conditio sine qua non relationship with degree of freedom (d. f.) of d. f. $=1$ is calculated as

$$
\begin{aligned}
\tilde{\chi}^{2} \text { Calculated }\left(A_{\mathrm{t}} \leftarrow B_{\mathrm{t}} \mid B\right) \equiv & \frac{(a-(a+c))^{2}}{B}+ \\
& \frac{((b+d)-\underline{B})^{2}}{\underline{B}} \\
& \equiv \frac{c^{2}}{B}+0 \\
& \equiv \frac{c^{2}}{B}
\end{aligned}
$$

or equally as

$$
\begin{aligned}
\tilde{\chi}^{2} \text { Calculated }\left(A_{\mathrm{t}} \leftarrow B_{\mathrm{t}} \mid \underline{A}\right) \equiv & \frac{(d-(c+d))^{2}}{\underline{A}}+ \\
& \frac{((a+b)-A)^{2}}{A} \\
& \frac{c^{2}}{\underline{A}}+0 \\
& \equiv \frac{c^{2}}{\underline{A}}
\end{aligned}
$$

and can be compared with a theoretical chi-square value at a certain level of significance $\alpha$. It has not yet been finally clarified whether the use of Yate's (Yates, 1934) continuity correction is necessary at all.

2.3.15. The left-tailed $p$ Value of the conditio sine qua non relationship

Definition 2.15 (The left-tailed p Value of the conditio sine qua non relationship).

The left-tailed (lt) p Value (Barukčić, 2019c) of the conditio sine qua non relationship can be calculated as follows.

$$
\begin{aligned}
\text { pValue }_{\mathrm{lt}}\left(A_{\mathrm{t}} \leftarrow B_{\mathrm{t}}\right) & \equiv 1-e^{-\left(1-p\left(A_{\mathrm{t}} \leftarrow B_{\mathrm{t}}\right)\right)} \\
& \equiv 1-e^{-(c / N)}
\end{aligned}
$$

\subsubsection{Sufficient condition}

\section{Definition 2.16 (Sufficient condition [Conditio per quam]).}

Mathematically, the sufficient condition (IMP) relationship, denoted by $p\left(A_{t} \rightarrow B_{t}\right)$ in terms of 
statistics and probability theory, is defined(Barukčić, 1989, p. 68-70) as

$$
\begin{aligned}
p\left(A_{\mathrm{t}} \rightarrow B_{\mathrm{t}}\right) \equiv p\left(\underline{\left.A_{\mathrm{t}} \vee B_{\mathrm{t}}\right)}\right. & \equiv \frac{\sum_{t=1}^{N}\left(\underline{A}_{\mathrm{t}} \vee B_{\mathrm{t}}\right)}{N} \equiv \frac{\left(\underline{A}_{\mathrm{t}} \vee B_{\mathrm{t}}\right) \times p\left(\underline{A}_{\mathrm{t}} \vee B_{\mathrm{t}}\right)}{\left(\underline{A}_{\mathrm{t}} \vee B_{\mathrm{t}}\right)} \\
& \equiv p\left(a_{\mathrm{t}}\right)+p\left(c_{\mathrm{t}}\right)+p\left(d_{\mathrm{t}}\right) \\
& \frac{N \times\left(p\left(a_{\mathrm{t}}\right)+p\left(c_{\mathrm{t}}\right)+p\left(d_{\mathrm{t}}\right)\right)}{N} \\
& \equiv \frac{a+c+d}{N} \equiv \frac{E\left(\underline{A_{\mathrm{t}}} \vee B_{\mathrm{t}}\right)}{N} \\
& \equiv \frac{B+d}{N} \equiv \frac{E\left(A_{\mathrm{t}} \rightarrow B_{\mathrm{t}}\right)}{N} \\
& \equiv \frac{a+\underline{A}}{N} \\
& \equiv+1
\end{aligned}
$$

It is $p\left(A_{\mathrm{t}}>B_{\mathrm{t}}\right) \equiv 1-p\left(A_{\mathrm{t}} \rightarrow B_{\mathrm{t}}\right)$ (see Table 11$)$.

Table 11. Sufficient condition.

\begin{tabular}{ccccc} 
& & \multicolumn{3}{c}{ Conditioned $\mathrm{B}_{\mathrm{t}}$} \\
& & TRUE & FALSE \\
\hline Condition & TRUE & $\mathrm{p}\left(\mathrm{a}_{\mathrm{t}}\right)$ & $\mathbf{+ 0}$ & $\mathrm{p}\left(\mathrm{A}_{\mathrm{t}}\right)$ \\
$\mathrm{A}_{\mathrm{t}}$ & FALSE & $\mathrm{p}\left(\mathrm{c}_{\mathrm{t}}\right)$ & $\mathrm{p}\left(\mathrm{d}_{\mathrm{t}}\right)$ & $\mathrm{p}\left(\underline{\mathrm{A}}_{\mathrm{t}}\right)$ \\
\hline & & $\mathrm{p}\left(\mathrm{B}_{\mathrm{t}}\right)$ & $\mathrm{p}\left(\underline{\mathrm{B}}_{\mathrm{t}}\right)$ & +1
\end{tabular}

Remark 2.4. A sufficient condition $A_{t}$ is characterized by the property that another event $B_{t}$ will occur if $A_{t}$ is given, if $A_{t}$ itself occured (Barukčić, 1989, 1997, 2005, 2016, 2017a,b, 2020a,b,c,d, Barukčić and Ufuoma, 2020). Example. The ground, the streets, the trees, human beings and many other objects too will become wet during heavy rain. Especially, if it is raining (event $A_{t}$ ), then human beings will become wet (event $\left.B_{t}\right)$. However, even if this is a common human wisdom, a human being equipped with an appropriate umbrella (denoted by $R_{t}$ ) need not become wet even during heavy rain. An appropriate umbrella $\left(R_{t}\right)$ is similar to an event with the potential to counteract the occurrence of another event $\left(B_{t}\right)$ and can be understood something as an anti-dot of another event. In other words, an appropriate umbrella is an antidote of the effect of rain on human body, an appropriate umbrella has the potential to protect humans from the effect of rain on their body. It is a good rule of thumb that the following relationship

$$
p\left(A_{t} \rightarrow B_{t}\right)+p\left(R_{t} \wedge B_{t}\right) \equiv+1
$$

indicates that $R_{t}$ is an antidote of $A_{t}$. However, taking a shower, swimming in a lake et cetera may make human hair wet too. More than anything else, however, these events does not affect the final outcome, the effect of raining on human body. 
2.3.17. The Chi square goodness of fit test of a sufficient condition relationship

\section{Definition 2.17 (The $\tilde{\chi}^{2}$ goodness of fit test of a sufficient condition relationship).}

Under some well known circumstances, testing hypothesis about the conditio per quam relationship $\mathrm{p}\left(\mathrm{A}_{\mathrm{t}} \rightarrow \mathrm{B}_{\mathrm{t}}\right)$ is possible by the chi-square distribution (also chi-squared or $\tilde{\chi}^{2}$-distribution) too. The $\tilde{\chi}^{2}$ goodness of fit test of a conditio per quam relationship with degree of freedom (d. f.) of d. f. $=1$ is calculated as

$$
\begin{aligned}
\tilde{\chi}_{\text {Calculated }}^{2}\left(A_{\mathrm{t}} \rightarrow B_{\mathrm{t}} \mid A\right) \equiv & \frac{(a-(a+b))^{2}}{A}+ \\
& \frac{((c+d)-\underline{A})^{2}}{\underline{A}} \\
& \equiv \frac{b^{2}}{A}+0 \\
& \equiv \frac{b^{2}}{A}
\end{aligned}
$$

or equally as

$$
\begin{aligned}
\tilde{\chi}^{2} \text { Calculated }\left(A_{\mathrm{t}} \rightarrow B_{\mathrm{t}} \mid \underline{B}\right) \equiv & \frac{(d-(b+d))^{2}}{\underline{B}}+ \\
& \frac{((a+c)-B)^{2}}{B} \\
& \frac{b^{2}}{\underline{B}}+0 \\
& \equiv \frac{b^{2}}{\underline{B}}
\end{aligned}
$$

and can be compared with a theoretical chi-square value at a certain level of significance $\alpha$. The $\tilde{\chi}^{2}$-distribution equals zero when the observed values are equal to the expected/theoretical values of the conditio per quam relationship/distribution $\mathrm{p}\left(\mathrm{A}_{\mathrm{t}} \rightarrow \mathrm{B}_{\mathrm{t}}\right)$, in which case the null hypothesis is accepted. Yate's (Yates, 1934) continuity correction has not been used in this context.

2.3.18. The left-tailed $\mathrm{p}$ Value of the conditio per quam relationship

\section{Definition 2.18 (The left-tailed p Value of the conditio per quam relationship).}

The left-tailed (lt) p Value (Barukčić, 2019c) of the conditio per quam relationship can be calculated as follows.

$$
\begin{aligned}
\operatorname{pValue}_{\mathrm{lt}}\left(A_{\mathrm{t}} \rightarrow B_{\mathrm{t}}\right) & \equiv 1-e^{-\left(1-p\left(A_{\mathrm{t}} \rightarrow B_{\mathrm{t}}\right)\right)} \\
& \equiv 1-e^{-(b / N)}
\end{aligned}
$$

Again, a low p-value indicates a statistical significance. 


\subsubsection{Necessary and sufficient conditions}

\section{Definition 2.19 (Necessary and sufficient conditions $[E Q V])$.}

The necessary and sufficient condition (EQV) relationship, denoted by $\mathrm{p}\left(\mathrm{A}_{t} \leftrightarrow \mathrm{B}_{t}\right)$ in terms of statistics and probability theory, is defined(Barukčić, 1989, p. 68-70) as

$$
\begin{aligned}
p\left(A_{\mathrm{t}} \leftrightarrow B_{\mathrm{t}}\right) & \equiv \frac{\sum_{t=1}^{N}\left(\left(A_{\mathrm{t}} \vee \underline{B}_{\mathrm{t}}\right) \wedge\left(\underline{A}_{\mathrm{t}} \vee B_{\mathrm{t}}\right)\right)}{N} \\
& \equiv p\left(a_{\mathrm{t}}\right)+p\left(d_{\mathrm{t}}\right) \\
& \equiv \frac{N \times\left(p\left(a_{\mathrm{t}}\right)+p\left(d_{\mathrm{t}}\right)\right)}{N} \\
& \equiv \frac{a+d}{N} \\
& \equiv+1
\end{aligned}
$$

2.3.20. The Chi square goodness of fit test of a necessary and sufficient condition relationship

\section{Definition 2.20 (The $\tilde{\chi}^{2}$ goodness of fit test of a necessary and sufficient condition relationship).}

Even the necessary and sufficient condition relationship $\mathrm{p}\left(\mathrm{A}_{\mathrm{t}} \leftrightarrow \mathrm{B}_{t}\right)$ can be tested by the chi-square distribution (also chi-squared or $\tilde{\chi}^{2}$-distribution) too. The $\tilde{\chi}^{2}$ goodness of fit test of a necessary and sufficient condition relationship with degree of freedom (d. f.) of d. f. $=1$ is calculated as

$$
\begin{aligned}
\tilde{\chi}^{2} \text { Calculated }\left(A_{\mathrm{t}} \leftrightarrow B_{\mathrm{t}} \mid A\right) \equiv & \frac{(a-(a+b))^{2}}{A}+ \\
& \frac{d-((c+d))^{2}}{\frac{A}{2}} \\
& \equiv \frac{b^{2}}{A}+\frac{c^{2}}{\underline{A}}
\end{aligned}
$$

or equally as

$$
\begin{aligned}
\tilde{\chi}_{\text {Calculated }}^{2}\left(A_{\mathrm{t}} \leftrightarrow B_{\mathrm{t}} \mid B\right) \equiv & \frac{(a-(a+c))^{2}}{B}+ \\
& \frac{d-((b+d))^{2}}{\underline{B}} \\
\equiv & \frac{c^{2}}{B}+\frac{b^{2}}{\underline{B}}
\end{aligned}
$$

The calculated $\tilde{\chi}^{2}$ goodness of fit test of a necessary and sufficient condition relationship can be compared with a theoretical chi-square value at a certain level of significance $\alpha$. Under conditions where the observed values are equal to the expected/theoretical values of a necessary and sufficient condition relationship/distribution $\mathrm{p}\left(\mathrm{A}_{\mathrm{t}} \leftrightarrow \mathrm{B}_{\mathrm{t}}\right)$, the $\tilde{\chi}^{2}$-distribution equals zero. It is to be cleared whether Yate's (Yates, 1934) continuity correction should be used at all. 
2.3.21. The left-tailed $p$ Value of a necessary and sufficient condition relationship

\section{Definition 2.21 (The left-tailed p Value of a necessary and sufficient condition relationship).}

The left-tailed (lt) p Value (Barukčić, 2019c) of a necessary and sufficient condition relationship can be calculated as follows.

$$
\begin{aligned}
\text { pValue }_{\mathrm{lt}}\left(A_{\mathrm{t}} \leftrightarrow B_{\mathrm{t}}\right) & \equiv 1-e^{-\left(1-p\left(A_{\mathrm{t}} \leftrightarrow B_{\mathrm{t}}\right)\right)} \\
& \equiv 1-e^{-((b+c) / N)}
\end{aligned}
$$

In this context, a low p-value indicates again a statistical significance. Table 12 may provide an overview of the theoretical distribution of a necessary and sufficient condition.

Table 12. Necessary and sufficient condition.

\begin{tabular}{lcccc}
\hline & \multicolumn{4}{c}{ Conditioned $\mathrm{B}_{\mathrm{t}}$} \\
& & YES & NO \\
\hline Condition $\mathrm{A}_{\mathrm{t}}$ & YES & 1 & 0 & 1 \\
& NO & 0 & 1 & 1 \\
\hline & & 1 & 1 & 2
\end{tabular}

\subsubsection{Either or conditions}

\section{Definition 2.22 (Either $A_{t}$ or $B_{t}$ conditions [NEQV]).}

Mathematically, an either $A_{t}$ or $B_{t}$ condition relationship (NEQV), denoted by $p\left(A_{t}>B_{t}\right)$ in terms of statistics and probability theory, is defined(Barukčić, 1989, p. 68-70) as

$$
\begin{aligned}
p\left(A_{\mathrm{t}}><B_{\mathrm{t}}\right) & \equiv \frac{\sum_{t=1}^{N}\left(\left(A_{\mathrm{t}} \wedge \underline{B}_{\mathrm{t}}\right) \vee\left(\underline{A}_{\mathrm{t}} \wedge B_{\mathrm{t}}\right)\right)}{N} \\
& \equiv p\left(b_{\mathrm{t}}\right)+p\left(c_{\mathrm{t}}\right) \\
& \equiv \frac{N \times\left(p\left(b_{\mathrm{t}}\right)+p\left(c_{\mathrm{t}}\right)\right)}{N} \\
& \equiv \frac{b+c}{N} \\
& \equiv+1
\end{aligned}
$$

It is $p\left(A_{\mathrm{t}}>B_{\mathrm{t}}\right) \equiv 1-p\left(A_{\mathrm{t}} \leftrightarrow B_{\mathrm{t}}\right)($ see Table 13).

Table 13. Either $A_{t}$ or $B_{t}$ relationship.

\begin{tabular}{lcccc}
\hline & \multicolumn{4}{c}{ Conditioned $\mathrm{B}_{\mathrm{t}}$} \\
& & YES & NO & \\
\hline Condition $\mathrm{A}_{\mathrm{t}}$ & YES & 0 & 1 & 1 \\
& NO & 1 & 0 & 1 \\
\hline & & 1 & 1 & 2
\end{tabular}


2.3.23. The Chi-square goodness of fit test of an either or condition relationship

Definition 2.23 (The $\tilde{\chi}^{2}$ goodness of fit test of an either or condition relationship).

An either or condition relationship $\mathrm{p}\left(\mathrm{A}_{\mathrm{t}}><\mathrm{B}_{\mathrm{t}}\right)$ can be tested by the chi-square distribution (also chi-squared or $\tilde{\chi}^{2}$-distribution) too. The $\tilde{\chi}^{2}$ goodness of fit test of an either or condition relationship with degree of freedom (d. f.) of d. f. $=1$ is calculated as

$$
\begin{aligned}
\tilde{\chi}^{2} \text { Calculated }\left(\left(A_{\mathrm{t}}><B_{\mathrm{t}}\right) \mid A\right) \equiv & \frac{(b-(a+b))^{2}}{A}+ \\
& \frac{c-((c+d))^{2}}{\frac{A}{2}} \\
& \equiv \frac{a^{2}}{A}+\frac{d^{2}}{\underline{A}}
\end{aligned}
$$

or equally as

$$
\begin{aligned}
\tilde{\chi}_{\text {Calculated }}^{2}\left(\left(A_{\mathrm{t}}><B_{\mathrm{t}}\right) \mid B\right) \equiv & \frac{(c-(a+c))^{2}}{B}+ \\
& \frac{b-((b+d))^{2}}{\underline{B}} \\
& \equiv \frac{a^{2}}{B}+\frac{d^{2}}{\underline{B}}
\end{aligned}
$$

Yate's (Yates, 1934) continuity correction has not been used in this context.

2.3.24. The left-tailed $\mathrm{p}$ Value of an either or condition relationship

\section{Definition 2.24 (The left-tailed p Value of an either or condition relationship).}

The left-tailed (lt) p Value (Barukčić, 2019c) of an either or condition relationship can be calculated as follows.

$$
\begin{aligned}
\text { pValue }_{\mathrm{lt}}\left(A_{\mathrm{t}}><B_{\mathrm{t}}\right) & \equiv 1-e^{-\left(1-p\left(A_{\mathrm{t}}>-<B_{\mathrm{t}}\right)\right)} \\
& \equiv 1-e^{-((a+d) / N)}
\end{aligned}
$$

In this context, a low p-value indicates again a statistical significance.

\subsubsection{Causal relationship $\mathrm{k}$}

\section{Definition 2.25 (Causal relationship k).}

Nonetheless, mathematically, the causal(Barukčić, 2011a,b, 2012) relationship (Barukčić, 1989, 1997, 2005, 2016, 2017b) between a cause $U_{t}$ (German: Ursache) and an effect $\mathrm{W}_{\mathrm{t}}$ (German: Wirkung), denoted by $\mathrm{k}\left(\mathrm{U}_{\mathrm{t}}, \mathrm{W}_{\mathrm{t}}\right)$, is defined at each single Bernoulli trial $t$ in terms of statistics and probability 
theory as

$$
\begin{aligned}
k\left(U_{\mathrm{t}}, W_{\mathrm{t}}\right) \equiv & \frac{\sigma\left(U_{\mathrm{t}}, W_{\mathrm{t}}\right)}{\sigma\left(U_{\mathrm{t}}\right) \times \sigma\left(W_{\mathrm{t}}\right)} \\
& \equiv \frac{p\left(U_{\mathrm{t}} \wedge W_{\mathrm{t}}\right)-p\left(U_{\mathrm{t}}\right) \times p\left(W_{\mathrm{t}}\right)}{\sqrt[2]{\left(p\left(U_{\mathrm{t}}\right) \times\left(1-p\left(U_{\mathrm{t}}\right)\right)\right) \times\left(p\left(W_{\mathrm{t}}\right) \times\left(1-p\left(W_{\mathrm{t}}\right)\right)\right)}}
\end{aligned}
$$

where $\sigma\left(\mathrm{U}_{\mathrm{t}}, \mathrm{W}_{\mathrm{t}}\right)$ denotes the co-variance between a cause $\mathrm{U}_{\mathrm{t}}$ and an effect $\mathrm{W}_{\mathrm{t}}$ at every single Bernoulli trial $t, \sigma\left(\mathrm{U}_{\mathrm{t}}\right)$ denotes the standard deviation of a cause $\mathrm{U}_{\mathrm{t}}$ at the same single Bernoulli trial $\mathrm{t}, \sigma\left(\mathrm{W}_{\mathrm{t}}\right)$ denotes the standard deviation of an effect $\mathrm{W}_{\mathrm{t}}$ at same single Bernoulli trial $\mathrm{t}$. Table 14 illustrates the theoretically possible relationships between a cause and an effect.

Table 14. Sample space and the causal relationship k

\begin{tabular}{ccccc} 
& & \multicolumn{3}{c}{ Effect $\mathrm{B}_{\mathrm{t}}$} \\
& & TRUE & FALSE \\
\hline Cause & TRUE & $\mathrm{p}\left(\mathrm{a}_{\mathrm{t}}\right)$ & $\mathrm{p}\left(\mathrm{b}_{\mathrm{t}}\right)$ & $\mathrm{p}\left(\mathrm{U}_{\mathrm{t}}\right)$ \\
$\mathrm{A}_{\mathrm{t}}$ & FALSE & $\mathrm{p}\left(\mathrm{c}_{\mathrm{t}}\right)$ & $\mathrm{p}\left(\mathrm{d}_{\mathrm{t}}\right)$ & $\mathrm{p}\left(\underline{\mathrm{U}}_{\mathrm{t}}\right)$ \\
\hline & & $\mathrm{p}\left(\mathrm{W}_{\mathrm{t}}\right)$ & $\mathrm{p}\left(\underline{\mathrm{W}}_{\mathrm{t}}\right)$ & +1
\end{tabular}

\subsubsection{Study design and bias}

Systematic observation and experimentation, inductive and deductive reasoning are essential for any formation and testing of hypotheses and theories about the natural world. In one way or another, logically and mathematically sound scientific methods and concepts are crucial constituents of any scientific progress. When all goes well, different scientists at different times and places using the same scientific methodology should be able to generate the same scientific knowledge. However, more than half $(52 \%)$ of scientists surveyed believe that studies do not successfully reproduce sufficiently similar or the same results as the original studies (Baker, 2016). In a very large study on publication bias in meta-analyses, Kicinski et al. (Kicinski et al., 2015) found evidence of publication bias even in systematic reviews. Therefore, a careful re-evaluation of the study/experimental design, the statistical methods and other scientific means which underpin scientific inquiry and research goals appears to be necessary once and again. While it is important to recognise the shortcoming of today's science, one issue which has shaped debates over studies published is the question: has a study really measured what it set out to? Even if studies carried out can vary greatly in detail, the data from the studies itself provide information about the credibility of the data.

\section{Index of unfairness (IOU)}

\section{Definition 2.26 (Index of unfairness).}

The index of unfairness (Barukčić, 2019b) (IOU) is defined as

$$
p(\operatorname{IOU}(A, B)) \equiv \text { Absolute }\left(\left(\frac{A+B}{N}\right)-1\right)
$$


A very good study design should assure as much as possible a $\mathrm{p}(\mathrm{IOU})=0$. In point of fact, against the background of lacking enough experience with the use of $p(I O U)$, a $p(I O U)$ up to 0.25 could be of use too. An index of unfairness is of use to prove whether sample data are biased and whether sample data can be used for Chi-square based analysis of necessary conditions, of sufficient conditions and of causal relationships.

\section{Index of independence (IOI)}

\section{Definition 2.27 (Index of independence).}

The index of independence(Barukčić, 2019a) (IOI) is defined as

$$
p(\operatorname{IOI}(A, \underline{B})) \equiv \text { Absolute }\left(\left(\frac{A+\underline{B}}{N}\right)-1\right)
$$

A very good study design which aims to prove an exclusion relationship or a causal relationship should assure as much as possible a $\mathrm{p}(\mathrm{IOI})=0$. However, once again, against the background of lacking enough experience with the use of $\mathrm{p}(\mathrm{IOI})$, sample data with a $\mathrm{p}(\mathrm{IOI})$ up to 0.25 are of use too. Today, most double-blind placebo-controlled studies are based on the demand that $\mathbf{p}(\mathbf{I O U})=\mathbf{p}($ IOI $)$ while the value of $\mathrm{p}(\mathrm{IOU})$ of has been widely neglected. Such an approach leads to unnecessary big sample sizes, the increase of cost, the waste of time and, most importantly of all, to epistemological systematically biased sample data and conclusions drawn. A change is necessary.

\section{Index of relationship (IOR)}

Definition 2.28 (Index of relationship (IOR)).

Due to several reasons, it is not always easy to identify the unique characteristics between two events like $A_{t}$ and $B_{t}$. And more than that, it is difficult to decide what to do, and much more difficult to know in which direction one should think and which decision is right. Sometimes it is helpful to know at least something about the direction of the relationship between two events like $\mathrm{A}_{\mathrm{t}}$ and $\mathrm{B}_{\mathrm{t}}$. Under conditions where $p\left(a_{\mathrm{t}}\right)=p\left(A_{\mathrm{t}} \wedge B_{\mathrm{t}}\right)$, the index of relationship(Barukčić, 2021a), abbreviated as IOR, is defined as

$$
\begin{aligned}
\operatorname{IOR}\left(A_{\mathrm{t}}, B_{\mathrm{t}}\right) & \equiv\left(\frac{p\left(A_{\mathrm{t}} \wedge B_{\mathrm{t}}\right)}{p\left(B_{\mathrm{t}}\right) \times p\left(A_{\mathrm{t}}\right)}\right)-1 \\
& \equiv\left(\frac{p\left(a_{\mathrm{t}}\right)}{p\left(B_{\mathrm{t}}\right) \times p\left(A_{\mathrm{t}}\right)}\right)-1 \\
& \equiv\left(\left(\frac{N \times N \times p\left(a_{\mathrm{t}}\right)}{N \times p\left(B_{\mathrm{t}}\right) \times N \times p\left(A_{\mathrm{t}}\right)}\right)-1\right) \\
& \equiv\left(\left(\frac{N \times a}{A \times B}\right)-1\right)
\end{aligned}
$$

where $p\left(A_{t}\right)$ denotes the probability of an event $A_{t}$ at the Bernoulli trial $t$ and $p\left(B_{t}\right)$ denotes the probability of another event $B_{t}$ at the same Bernoulli trial $t$ while $p\left(a_{t}\right)$ denotes the joint probability of $\mathrm{p}\left(\mathrm{A}_{\mathrm{t}}\right.$ AND $\left.\mathrm{B}_{\mathrm{t}}\right)$ at the same Bernoulli trial $\mathrm{t}$ and $\mathrm{a}, \mathrm{A}$ and $\mathrm{B}$ may denote the expectation values. 
2.3.27. Relative risk (RR)

\section{Relative risk $\left(\mathbf{R R}_{\mathbf{n c}}\right)$}

Definition 2.29 (Relative risk $\left(\mathrm{RR}_{\mathrm{nc}}\right)$ ).

The degree of association between the two binomial variables can be assessed by a number of very different coefficients, the relative (Cornfield, 1951, Sadowsky et al., 1953) risk is one(Barukčić, 2021c) of them. In general, relative risk $R_{\mathrm{nc}}$, which provides some evidence of a necessary condition, is defined as

$$
R R\left(A_{\mathrm{t}}, B_{\mathrm{t}}\right)_{\mathrm{nc}}=\frac{\frac{p\left(a_{\mathrm{t}}\right)}{p\left(A_{\mathrm{t}}\right)}}{\frac{p\left(c_{\mathrm{t}}\right)}{p\left(N o t A_{\mathrm{t}}\right)}}=\frac{p\left(a_{\mathrm{t}}\right) \times p\left(N o t A_{\mathrm{t}}\right)}{p\left(c_{\mathrm{t}}\right) \times p\left(A_{\mathrm{t}}\right)}=\frac{N \times p\left(a_{\mathrm{t}}\right) \times N \times p\left(N o t A_{\mathrm{t}}\right)}{N \times p\left(c_{\mathrm{t}}\right) \times N \times p\left(A_{\mathrm{t}}\right)}=\frac{a_{\mathrm{t}} \times\left(N o t A_{\mathrm{t}}\right)}{c_{\mathrm{t}} \times A_{\mathrm{t}}}=\frac{E E R\left(A_{\mathrm{t}}, B_{\mathrm{t}}\right)}{C E R\left(A_{\mathrm{t}}, B_{\mathrm{t}}\right)}
$$

That what scientist generally understand by relative risk is the ratio of a probability of an event occurring with an exposure versus the probability of an event occurring without an exposure. In other words,

relative risk $=($ probability $($ event in exposed group $)) /($ probability $($ the same event in not exposed group)).

A RR $\left(A_{t}, B_{t}\right)=+1$ means that exposure does not affect the outcome or both are independent of each other while $\operatorname{RR}\left(A_{t}, B_{t}\right)$ less than +1 means that the risk of the outcome is decreased by the exposure. In this context, an $\operatorname{RR}\left(\mathrm{A}_{t}, \mathrm{~B}_{\mathrm{t}}\right)$ greater than +1 denotes that the risk of the outcome is increased by the exposure. Widely known problems with odds ratio and relative risk are already documented in literature.

\section{Relative risk ( $\mathbf{R R}(\mathbf{s c}))$}

Definition 2.30 (Relative risk (RR (sc))).

The relative risk (sc), which provides some evidence of a sufficient condition, is calculated from the point of view of an outcome and is defined as

$$
R R\left(A_{\mathrm{t}}, B_{\mathrm{t}}\right)_{\mathrm{sc}}=\frac{\frac{p\left(a_{\mathrm{t}}\right)}{p\left(B_{\mathrm{t}}\right)}}{\frac{p\left(b_{\mathrm{t}}\right)}{p\left(N o t B_{\mathrm{t}}\right)}}=\frac{p\left(a_{\mathrm{t}}\right) \times p\left(N o t B_{\mathrm{t}}\right)}{p\left(b_{\mathrm{t}}\right) \times p\left(B_{\mathrm{t}}\right)}=\frac{N \times p\left(a_{\mathrm{t}}\right) \times N \times p\left(N o t B_{\mathrm{t}}\right)}{N \times p\left(b_{\mathrm{t}}\right) \times N \times p\left(B_{\mathrm{t}}\right)}=\frac{a_{\mathrm{t}} \times\left(N o t B_{\mathrm{t}}\right)}{b_{\mathrm{t}} \times B_{\mathrm{t}}}=\frac{O P R\left(A_{\mathrm{t}}, B_{\mathrm{t}}\right)}{C P R\left(A_{\mathrm{t}}, B_{\mathrm{t}}\right)}
$$

\section{Relative risk reduction ( $R R R)$}

Definition 2.31 (Relative risk reduction (RRR)). 


$$
\begin{aligned}
\operatorname{RRR}\left(A_{\mathrm{t}}, B_{\mathrm{t}}\right) & \equiv \frac{\operatorname{CER}\left(A_{\mathrm{t}}, B_{\mathrm{t}}\right)-\operatorname{EER}\left(A_{\mathrm{t}}, B_{\mathrm{t}}\right)}{\operatorname{CER}\left(A_{\mathrm{t}}, B_{\mathrm{t}}\right)} \\
& =1-\operatorname{RR}\left(A_{\mathrm{t}}, B_{\mathrm{t}}\right)
\end{aligned}
$$

\section{Vaccine efficacy (VE)}

Definition 2.32 (Vaccine efficacy (VE)).

Vaccine efficacy is defined as the percentage reduction of a disease in a vaccinated group of people as compared to an unvaccinated group of people.

$$
\begin{aligned}
V E\left(A_{\mathrm{t}}, B_{\mathrm{t}}\right) & \equiv 100 \times\left(1-R R\left(A_{\mathrm{t}}, B_{\mathrm{t}}\right)\right) \\
& \equiv 100 \times\left(\frac{C E R\left(A_{\mathrm{t}}, B_{\mathrm{t}}\right)-E E R\left(A_{\mathrm{t}}, B_{\mathrm{t}}\right)}{C E R\left(A_{\mathrm{t}}, B_{\mathrm{t}}\right)}\right)
\end{aligned}
$$

Historically, vaccine efficacy has been designed to evaluate the efficacy of a certain vaccine by Greenwood and Yule in 1915 for the cholera and typhoid vaccines(Greenwood and Yule, 1915) and best measured using double-blind, randomized, clinical controlled trials. However, the calculated vaccine efficacy is depending too much on the study design, can lead to erroneous conclusions and is only of very limited value.

\section{Experimental event rate (EER)}

Definition 2.33 (Experimental event rate (EER)).

$$
\operatorname{EER}\left(A_{\mathrm{t}}, B_{\mathrm{t}}\right) \equiv \frac{p\left(a_{\mathrm{t}}\right)}{p\left(A_{\mathrm{t}}\right)}=\frac{a_{\mathrm{t}}}{a_{\mathrm{t}}+b_{\mathrm{t}}}
$$

Definition 2.34 (Control event rate (CER)).

$$
\operatorname{CER}\left(A_{\mathrm{t}}, B_{\mathrm{t}}\right) \equiv \frac{p\left(c_{\mathrm{t}}\right)}{p\left(\underline{A_{\mathrm{t}}}\right)}=\frac{c_{\mathrm{t}}}{c_{\mathrm{t}}+d_{\mathrm{t}}}
$$


Absolute risk reduction (ARR)

Definition 2.35 (Absolute risk reducation (ARR)).

$$
\begin{aligned}
\operatorname{ARR}\left(A_{\mathrm{t}}, B_{\mathrm{t}}\right) & \equiv \frac{p\left(c_{\mathrm{t}}\right)}{p\left(\underline{A_{\mathrm{t}}}\right)}-\frac{p\left(a_{\mathrm{t}}\right)}{p\left(A_{\mathrm{t}}\right)} \\
& =\frac{c_{\mathrm{t}}}{c_{\mathrm{t}}+d_{\mathrm{t}}}-\frac{a_{\mathrm{t}}}{a_{\mathrm{t}}+b_{\mathrm{t}}} \\
& =\operatorname{CER}\left(A_{\mathrm{t}}, B_{\mathrm{t}}\right)-\operatorname{EER}\left(A_{\mathrm{t}}, B_{\mathrm{t}}\right)
\end{aligned}
$$

\section{Absolute risk increase (ARI)}

Definition 2.36 (Absolute risk increase (ARI)).

$$
\begin{aligned}
\operatorname{ARI}\left(A_{\mathrm{t}}, B_{\mathrm{t}}\right) & \equiv \frac{p\left(a_{\mathrm{t}}\right)}{p\left(A_{\mathrm{t}}\right)}-\frac{p\left(c_{\mathrm{t}}\right)}{p\left(\underline{A}_{\mathrm{t}}\right)} \\
& =\operatorname{EER}\left(A_{\mathrm{t}}, B_{\mathrm{t}}\right)-\operatorname{CER}\left(A_{\mathrm{t}}, B_{\mathrm{t}}\right)
\end{aligned}
$$

\section{Number needed to treat (NNT)}

Definition 2.37 (Number needed to treat (NNT)).

$$
\operatorname{NNT}\left(A_{\mathrm{t}}, B_{\mathrm{t}}\right) \equiv \frac{1}{\operatorname{CER}\left(A_{\mathrm{t}}, B_{\mathrm{t}}\right)-\operatorname{EER}\left(A_{\mathrm{t}}, B_{\mathrm{t}}\right)}
$$

An ideal number needed to treat(Cook and Sackett, 1995, Laupacis et al., 1988), mathematically the reciprocal of the absolute risk reduction, is NNT $=1$. Under these circumstances, everyone improves with a treatment, while no one improves with control. A higher number needed to treat indicates more or less a treatment which is less effective.

\section{Number needed to harm (NNH)}

Definition 2.38 (Number needed to harm $(\mathrm{NNH})$ ).

$$
\operatorname{NNH}\left(A_{\mathrm{t}}, B_{\mathrm{t}}\right) \equiv \frac{1}{\operatorname{EER}\left(A_{\mathrm{t}}, B_{\mathrm{t}}\right)-\operatorname{CER}\left(A_{\mathrm{t}}, B_{\mathrm{t}}\right)}
$$

The number needed to harm (Massel and Cruickshank, 2002), mathematically the inverse of the absolute risk increase, indicates at the end how many patients need to be exposed to a certain factor, in order to observe a harm in one patient that would not otherwise have been harmed. 
Outcome prevalence rate (OPR)

Definition 2.39 (Outcome prevalence rate (OPR)).

$$
\operatorname{OPR}\left(A_{\mathrm{t}}, B_{\mathrm{t}}\right) \equiv \frac{p\left(a_{\mathrm{t}}\right)}{p\left(B_{\mathrm{t}}\right)}=\frac{a_{\mathrm{t}}}{a_{\mathrm{t}}+c_{\mathrm{t}}}
$$

\section{Control prevalence rate $(\mathrm{CPR})$}

Definition 2.40 (Control prevalence rate (CPR)).

$$
C P R\left(A_{\mathrm{t}}, B_{\mathrm{t}}\right) \equiv \frac{p\left(b_{\mathrm{t}}\right)}{p\left(\underline{B}_{\mathrm{t}}\right)}=\frac{b_{\mathrm{t}}}{b_{\mathrm{t}}+d_{\mathrm{t}}}
$$

Bias and confounding is present to some degree in all research. In order to assess the relationship of exposure with a disease or an outcome, a fictive control group (i.e. of newborn or of young children et cetera) can be of use too. Under certain circumstances, even a CPR $=0$ is imaginable.

\section{Absolute prevalence reduction (APR)}

Definition 2.41 (Absolute prevalence reduction (APR)).

$$
\operatorname{APR}\left(A_{\mathrm{t}}, B_{\mathrm{t}}\right) \equiv C P R\left(A_{\mathrm{t}}, B_{\mathrm{t}}\right)-O P R\left(A_{\mathrm{t}}, B_{\mathrm{t}}\right)
$$

\section{Absolute prevalence increase (API)}

Definition 2.42 (Absolute prevalence increase (API)).

$$
A P I\left(A_{\mathrm{t}}, B_{\mathrm{t}}\right) \equiv O P R\left(A_{\mathrm{t}}, B_{\mathrm{t}}\right)-C P R\left(A_{\mathrm{t}}, B_{\mathrm{t}}\right)
$$

\section{Relative prevalence reduction (RPR)}

Definition 2.43 (Relative prevalence reduction (RPR)).

$$
\begin{aligned}
\operatorname{RPR}\left(A_{\mathrm{t}}, B_{\mathrm{t}}\right) & \equiv \frac{C P R\left(A_{\mathrm{t}}, B_{\mathrm{t}}\right)-O P R\left(A_{\mathrm{t}}, B_{\mathrm{t}}\right)}{\operatorname{CPR}\left(A_{\mathrm{t}}, B_{\mathrm{t}}\right)} \\
& =1-R R\left(A_{\mathrm{t}}, B_{\mathrm{t}}\right)_{\mathrm{sc}}
\end{aligned}
$$


The index NNS

Definition 2.44 (The index NNS).

$$
\operatorname{NNS}\left(A_{\mathrm{t}}, B_{\mathrm{t}}\right) \equiv \frac{1}{\operatorname{CPR}\left(A_{\mathrm{t}}, B_{\mathrm{t}}\right)-O P R\left(A_{\mathrm{t}}, B_{\mathrm{t}}\right)}
$$

Mathematically, the index NNS is the reciprocal of the absolute prevalence reduction.

\section{The index NNI}

Definition 2.45 (The index NNI).

$$
\operatorname{NNI}\left(A_{\mathrm{t}}, B_{\mathrm{t}}\right) \equiv \frac{1}{O P R\left(A_{\mathrm{t}}, B_{\mathrm{t}}\right)-C P R\left(A_{\mathrm{t}}, B_{\mathrm{t}}\right)}
$$

Mathematically, the index NNI is the reciprocal of the absolute prevalence increase.

\subsubsection{Odds ratio $(\mathrm{OR})$}

Definition 2.46 (Odds ratio (OR)).

Odds ratios as an appropriate measure for estimating the relative risk have become widely used in medical reports of case-control studies. The odds ratio(Fisher, 1935, p. 50) is defined(Cox, 1958) as the ratio of the odds of an event occurring in one group with respect to the odds of its occurring in another group. Odds(Yule and Pearson, 1900b, p. 273) ratio (OR) is a measure of association which quantifies the relationship between two binomial distributed random variables (exposure vs. outcome) and is related to Yule's (Yule and Pearson, 1900b, p. 272) Q(Yule, 1912, p. 585/586). Two events $A_{t}$ and $\mathrm{B}_{\mathrm{t}}$ are regarded as independent if $\left(A_{\mathrm{t}}, B_{\mathrm{t}}\right)=1$. Let

$a_{t}=$ number of persons exposed to $A_{t}$ and with disease $B_{t}$

$b_{t}=$ number of persons exposed to $A_{t}$ but without disease $\underline{B}_{t}$

$c_{t}=$ number of persons unexposed $\underline{A}_{t}$ but with disease $B_{t}$

$\mathrm{d}_{\mathrm{t}}=$ number of persons unexposed $\underline{A}_{t}$ : and without disease $\underline{B}_{t}$

$a_{t}+c_{t}=$ total number of persons with disease $B_{t}$ (case-patients)

$\mathrm{b}_{\mathrm{t}}+\mathrm{d}_{\mathrm{t}}=$ total number of persons without disease $\underline{B}_{\mathrm{t}}$ (controls).

Hereafter, consider the table 15 . The odds' ratio (OR) is defined as

$$
\begin{aligned}
O R\left(A_{\mathrm{t}}, B_{\mathrm{t}}\right) & \equiv\left(\frac{a_{\mathrm{t}}}{b_{\mathrm{t}}}\right) /\left(\frac{c_{\mathrm{t}}}{d_{\mathrm{t}}}\right) \\
& \equiv\left(\frac{a_{\mathrm{t}} \times d_{\mathrm{t}}}{b_{\mathrm{t}} \times c_{\mathrm{t}}}\right)
\end{aligned}
$$

Remark 2.5. Odds ratios can support logical fallacies and cause difficulties in drawing logically consistent conclusions. The chorus of voices is growing, which demand the immediate ending(Knol, 2012, Sackett et al., 1996) of any use of Odds ratio. 
Table 15. The two by two table of random variables

\begin{tabular}{ccccc} 
& \multicolumn{4}{c}{ Conditioned/Outcome $\mathrm{B}_{\mathrm{t}}$} \\
& & TRUE & FALSE & \\
\hline Condition/Exposure & TRUE & $\mathrm{a}_{\mathrm{t}}$ & $\mathrm{b}_{\mathrm{t}}$ & $\mathrm{A}_{\mathrm{t}}$ \\
$\mathrm{A}_{\mathrm{t}}$ & FALSE & $\mathrm{c}_{\mathrm{t}}$ & $\mathrm{d}_{\mathrm{t}}$ & $\underline{\mathrm{A}}_{\mathrm{t}}$ \\
\hline & & $\mathrm{B}_{\mathrm{t}}$ & $\underline{\mathrm{B}}_{\mathrm{t}}$ & $\mathrm{N}_{\mathrm{t}}$
\end{tabular}

Under conditions where $(b=0)$, the measure of association odds ratio will collapse, because we need to divide by zero, as can be seen at eq. 2.80. However, according to today's rules of mathematics, a division by zero is neither allowed nor generally accepted as possible. It does no harm to remind ourselves that in the case $b=0$ the event $A_{t}$ is a sufficient condition of $B_{t}$. In other words, odds ratio is not able to recognize elementary relationships of objective reality. In fact, it would be a failure not to recognize how dangerous and less valuable odds ratio is.

Under conditions where $(c=0)$ odds ratio collapses too, because we need again to divide by zero, as can be seen at eq. 2.80. However, and again, today's rules of mathematics don't allow us a division by zero. In point of fact, in the case $c=0$ it is more than necessary to point out that $A_{t}$ is a necessary condition of $B_{t}$. In other words, odds ratio or the cross-product ratio is not able to recognize elementary relationships of nature like necessary conditions. We can and need to overcome all the epistemological obstacles as backed by odds ratio entirety. Sooner rather than later, we should give up this measure of relationship completely.

\subsection{Statistical methods}

The probability of the necessary (Barukčić, 2021b) condition $\mathrm{p}(\mathrm{SINE})$ has been calculated and tested for statistical significance. The probability of the sufficient (Barukčić, 2021b) condition p(IMP) has been calculated, the statistical significance of this relationship has been proofed. The chi-square goodness of fit test with one degree of freedom has been used to test whether the sample data published fit a certain theoretical distribution in the population. The causal relationship k (Barukčić, 2021b) has been calculated to evaluate a possible causal relationship between the events/factors analysed. The hyper-geometric(Fisher, 1922, Gonin, 1936, Huygens and van Schooten, 1657, Pearson, 1899) distribution (HGD) has been used to test the one-sided significance of the causal relationship k. The study (design) bias has been controlled by IOI, the index of independence(Barukčić, 2019a) and IOU, the index of unfairness(Barukčić, 2019b). All the data were analysed using MS Excel (Microsoft Corporation, USA). The $\mathrm{p}$ values less than 0.05 were considered to indicate a statistically significant difference. 
2.5. Axioms

2.5.1. Axiom I. Lex identitatis

In this context, we define axiom I as the expression

$$
+1=+1
$$

2.5.2. Axiom II. Lex contradictionis

In this context, axiom II or lex contradictionis, the negative of lex identitatis, or

$$
+0=+1
$$

and equally the most simple form of a contradiction formulated.

2.5.3. Axiom III. Lex negationis

$$
\neg(0) \times 0=1
$$

where $\neg$ denotes (logical (Boole, 1854) or natural) negation (Ayer, 1952, Förster and Melamed, 2012, Hedwig, 1980, Heinemann, 1943, Horn, 1989, Koch, 1999, Kunen, 1987, Newstadt, 2015, Royce, 1917, Speranza and Horn, 2010, Wedin, 1990). In this context, there is some evidence that $\neg(1) \times 1=0$. In other words, it is $(\neg(1) \times 1) \times(\neg(0) \times 0)=1$ 


\section{Results}

Theorem 3.1. In general, the mutually exclusive relationship is defined as

$$
p\left(A_{t} \mid B_{t}\right) \equiv p\left(A_{t} \uparrow B_{t}\right) \equiv \frac{b+\underline{A}}{N} \equiv \frac{c+\underline{B}}{N} \equiv+1
$$

Proof by direct proof. The premise

$$
+1 \equiv+1
$$

is true. In the following, we rearrange the premise. We obtain

$$
N \equiv N
$$

and equally

$$
a+b+c+d \equiv a+b+c+d \equiv N
$$

Mutually exclusive events (at a certain (period of) time t) or the exclusion relationship is defined by the condition that the joint distribution function $p\left(A_{\mathrm{t}} \cap B_{\mathrm{t}}\right)=0$ or that $\mathrm{a}=0$. Equation 3.4 becomes

$$
b+(c+d) \equiv c+(b+d) \equiv N
$$

or

$$
b+\underline{A} \equiv c+\underline{B} \equiv N
$$

or

$$
\frac{b+\underline{A}}{N} \equiv \frac{c+\underline{B}}{N} \equiv \frac{N}{N} \equiv+1
$$

In general, the exclusion relationship or mutually exclusive events are determined by the equation

$$
p\left(A_{\mathrm{t}} \mid B_{\mathrm{t}}\right) \equiv p\left(A_{\mathrm{t}} \uparrow B_{\mathrm{t}}\right) \equiv \frac{b+\underline{A}}{N} \equiv \frac{c+\underline{B}}{N} \equiv+1
$$

Table 8 illustrated this relationship in more detail.

\subsection{Mutually exclusive events and relative risk}

Theorem 3.2 (Mutually exclusive events and relative risk). In general, a relative risk(see Cornfield, 1951, Sadowsky et al., 1953) which is equal to

$$
R R\left(A_{t}, B_{t}\right)_{n c} \equiv 0
$$

is indicating mutually exclusive events (at a certain (period of) time t) or the exclusion relationship. Proof by direct proof. The premise

$$
+1 \equiv+1
$$

is true. In the following, we rearrange the premise. The relative risk $R R\left(A_{t}, B_{t}\right)_{n c}$ is equal to itself. It is

$$
R R\left(A_{\mathrm{t}}, B_{\mathrm{t}}\right)_{\mathrm{nc}} \equiv R R\left(A_{\mathrm{t}}, B_{\mathrm{t}}\right)_{\mathrm{nc}}
$$


or

$$
R R\left(A_{\mathrm{t}}, B_{\mathrm{t}}\right)_{\mathrm{nc}} \equiv \frac{p\left(a_{\mathrm{t}}\right) \times p\left(N o t A_{\mathrm{t}}\right)}{p\left(A_{\mathrm{t}}\right) \times p\left(c_{\mathrm{t}}\right)}
$$

Mutually exclusive events (at a certain (period of) time $t$ ) or the exclusion relationship itself are determined by the condition that the joint distribution function $p\left(A_{\mathrm{t}} \cap B_{\mathrm{t}}\right)=0$ or that $\mathrm{a}_{\mathrm{t}}=0$. Equation 3.12 becomes

$$
R R\left(A_{\mathrm{t}}, B_{\mathrm{t}}\right)_{\mathrm{nc}} \equiv \frac{0 \times p\left(N o t A_{\mathrm{t}}\right)}{p\left(A_{\mathrm{t}}\right) \times p\left(c_{\mathrm{t}}\right)}
$$

Mutually exclusive events (at a certain (period of) time $t$ ) or the exclusion relationship are indicated by a relative risk

$$
R R\left(A_{\mathrm{t}}, B_{\mathrm{t}}\right)_{\mathrm{nc}} \equiv 0
$$

\subsection{Mutually exclusive events and odds ratio}

Theorem 3.3. In general, an

$$
\operatorname{OR}\left(A_{t}, B_{t}\right) \equiv 0
$$

is indicating mutually exclusive events (at a certain (period of) time t) or an exclusion relationship between events which occur at the same (period of) time / Bernoulli trial (see Uspensky, 1937) t.

Proof by direct proof. The premise

$$
+1 \equiv+1
$$

is true. $\mathrm{OR}\left(\mathrm{A}_{t}, \mathrm{~B}_{t}\right)$, the odds(see also Fisher, 1935) ratio, is another measure of association(see also Yule and Pearson, 1900a, p. 273) which quantifies the relationship between two (binomial distributed) random variables (exposure vs. outcome) and is related to Yule's (see also Yule and Pearson, 1900a, p. 272) Q(see also Yule, 1912, p. 585/586). In the following, we rearrange the premise. We obtain

$$
\operatorname{OR}\left(A_{\mathrm{t}}, B_{\mathrm{t}}\right) \equiv O R\left(A_{\mathrm{t}}, B_{\mathrm{t}}\right)
$$

or

$$
\operatorname{OR}\left(A_{\mathrm{t}}, B_{\mathrm{t}}\right) \equiv\left(\frac{a_{\mathrm{t}} \times d_{\mathrm{t}}}{b_{\mathrm{t}} \times c_{\mathrm{t}}}\right)
$$

Mutually exclusive events (at a certain (period of) time $\mathrm{t}$ ) or the exclusion relationship itself are determined by the condition that the joint distribution function $p\left(A_{\mathrm{t}} \cap B_{\mathrm{t}}\right)=0$ or that $\mathrm{a}_{\mathrm{t}}=0$. Equation 3.18 becomes

$$
\operatorname{OR}\left(A_{\mathrm{t}}, B_{\mathrm{t}}\right) \equiv\left(\frac{0 \times d_{\mathrm{t}}}{b_{\mathrm{t}} \times c_{\mathrm{t}}}\right)
$$

Mutually exclusive events (at a certain (period of) time $t$ ) or the exclusion relationship are indicated by an odds ratio

$$
\operatorname{OR}\left(A_{\mathrm{t}}, B_{\mathrm{t}}\right) \equiv 0
$$




\subsection{Approximation of mutually exclusive events}

Placebo controlled randomized trials are controversial, ethically and mathematically. Withholding a valuable treatment poses unnecessary risks and serious harm to participants, while the study design of placebo controlled randomized trials requires a sample size which seems pointless. Mathematically, it is possible to estimate the effect of an event $A_{t}$ on an outcome $B_{t}$.

Theorem 3.4. In general, the exclusion relationship follows approximately as

$$
p\left(A_{t} \mid B_{t}\right) \equiv p\left(A_{t} \uparrow B_{t}\right) \approx 1-\frac{p\left(a_{t}\right)}{p\left(A_{t}\right)}
$$

Proof by direct proof. The premise

$$
+1 \equiv+1
$$

is true. In the following, we rearrange the premise. We obtain

$$
p\left(A_{\mathrm{t}}\right) \equiv p\left(A_{\mathrm{t}}\right)
$$

or

$$
p\left(a_{\mathrm{t}}\right)+p\left(b_{\mathrm{t}}\right) \equiv p\left(A_{\mathrm{t}}\right)
$$

Rearranging equation 3.24 , it is

$$
p\left(b_{\mathrm{t}}\right) \equiv p\left(A_{\mathrm{t}}\right)-p\left(a_{\mathrm{t}}\right)
$$

Simplifying equation 3.25 , we obtain

$$
\frac{p\left(b_{\mathrm{t}}\right)}{p\left(A_{\mathrm{t}}\right)} \equiv \frac{p\left(A_{\mathrm{t}}\right)}{p\left(A_{\mathrm{t}}\right)}-\frac{p\left(a_{\mathrm{t}}\right)}{p\left(A_{\mathrm{t}}\right)}
$$

Equation 3.26 becomes

$$
\frac{p\left(b_{\mathrm{t}}\right)}{p\left(A_{\mathrm{t}}\right)} \equiv 1-\frac{p\left(a_{\mathrm{t}}\right)}{p\left(A_{\mathrm{t}}\right)}
$$

Mutually exclusive events demand that $\frac{p\left(b_{\mathrm{t}}\right)}{p\left(A_{\mathrm{t}}\right)} \equiv 1$ which is not given under any circumstances. Therefore, the exclusion relationship can only be estimated roughly by the relationship

$$
p\left(A_{\mathrm{t}} \mid B_{\mathrm{t}}\right) \equiv p\left(A_{\mathrm{t}} \uparrow B_{\mathrm{t}}\right) \approx 1-\frac{p\left(a_{\mathrm{t}}\right)}{p\left(A_{\mathrm{t}}\right)}
$$

In reality, the exclusion relationship will be stronger than suggested by the equation 3.28. Therefore, equation 3.28 is of particular value under conditions where a placebo group is absent or appears to be completely unsuitable. 


\subsection{Approximation of mutually exclusive events}

Case-control studies or retrospective ${ }^{22}$ studies are observational studies which can contribute to the identification of risk factors, conditions and causes of disease or changes in general. Due to the study design, these studies are particularly susceptible to generate various forms of bias. Therefore, a rough and reliable estimate of a certain relationship like the exclusion relationship which is independent of the study design bias appears to be helpful.

Theorem 3.5. In general, the exclusion relationship follows approximately as

$$
p\left(A_{t} \mid B_{t}\right) \equiv p\left(A_{t} \uparrow B_{t}\right) \approx 1-\frac{p\left(a_{t}\right)}{p\left(B_{t}\right)}
$$

Proof by direct proof. The premise

$$
+1 \equiv+1
$$

is true. In the following, we rearrange the premise. We obtain

$$
p\left(B_{\mathrm{t}}\right) \equiv p\left(B_{\mathrm{t}}\right)
$$

where $p\left(B_{\mathrm{t}}\right)$ is the probability of an outcome $\mathrm{B}_{\mathrm{t}}$ within a sample or a population. It is

$$
p\left(a_{\mathrm{t}}\right)+p\left(c_{\mathrm{t}}\right) \equiv p\left(B_{\mathrm{t}}\right)
$$

Rearranging equation 3.32 , it is

$$
p\left(c_{\mathrm{t}}\right) \equiv p\left(B_{\mathrm{t}}\right)-p\left(a_{\mathrm{t}}\right)
$$

Simplifying equation 3.33, we obtain

$$
\frac{p\left(c_{\mathrm{t}}\right)}{p\left(B_{\mathrm{t}}\right)} \equiv \frac{p\left(B_{\mathrm{t}}\right)}{p\left(B_{\mathrm{t}}\right)}-\frac{p\left(a_{\mathrm{t}}\right)}{p\left(B_{\mathrm{t}}\right)}
$$

Equation 3.34 becomes

$$
\frac{p\left(c_{\mathrm{t}}\right)}{p\left(B_{\mathrm{t}}\right)} \equiv 1-\frac{p\left(a_{\mathrm{t}}\right)}{p\left(B_{\mathrm{t}}\right)}
$$

Mutually exclusive events demand that $\frac{p\left(c_{\mathrm{t}}\right)}{p\left(B_{\mathrm{t}}\right)} \equiv 1$ which is not given under any circumstances. Therefore, the exclusion relationship can only be estimated roughly by the relationship

$$
p\left(A_{\mathrm{t}} \mid B_{\mathrm{t}}\right) \equiv p\left(A_{\mathrm{t}} \uparrow B_{\mathrm{t}}\right) \approx 1-\frac{p\left(a_{\mathrm{t}}\right)}{p\left(B_{\mathrm{t}}\right)}
$$

\footnotetext{
${ }^{22}$ PMID: 20299809
} 


\subsection{Very conservative approximation of mutually exclusive events}

In reality, the exclusion relationship will be stronger than suggested by the equation 3.36. Therefore, equation 3.36 is of particular value under conditions where a control group is absent or appears to be completely unsuitable.

Theorem 3.6 (Very conservative approximation of mutually exclusive events). In general, a rough and only approximate estimate of the mutually exclusive relationship is given by the equation

$$
p\left(A_{t} \mid B_{t}\right) \geq+1-\frac{p\left(a_{t}\right)}{p\left(A_{t}\right)}
$$

Proof by direct proof. The premise

$$
+1 \geq p\left(A_{\mathrm{t}}\right)
$$

is true. In the following, we rearrange the premise. We obtain

$$
p\left(a_{\mathrm{t}}\right) \geq p\left(a_{\mathrm{t}}\right) \times p\left(A_{\mathrm{t}}\right)
$$

Dividing equation 3.39 by $p\left(A_{\mathrm{t}}\right)$, it is

$$
\frac{p\left(a_{\mathrm{t}}\right)}{p\left(A_{\mathrm{t}}\right)} \geq \frac{p\left(a_{\mathrm{t}}\right) \times p\left(A_{\mathrm{t}}\right)}{p\left(A_{\mathrm{t}}\right)}
$$

or

$$
\frac{p\left(a_{\mathrm{t}}\right)}{p\left(A_{\mathrm{t}}\right)} \geq p\left(a_{\mathrm{t}}\right)
$$

while $p\left(a_{\mathrm{t}}\right)=p\left(A_{\mathrm{t}} \cap B_{\mathrm{t}}\right)$ is the joint probability or distribution et cetera of $A_{\mathrm{t}} \cap B_{\mathrm{t}}$. Adding $p\left(b_{\mathrm{t}}\right)$ to equation 3.41 , it is

$$
p\left(b_{\mathrm{t}}\right)+\frac{p\left(a_{\mathrm{t}}\right)}{p\left(A_{\mathrm{t}}\right)} \geq p\left(a_{\mathrm{t}}\right)+p\left(b_{\mathrm{t}}\right)
$$

or

$$
p\left(b_{\mathrm{t}}\right)+\frac{p\left(a_{\mathrm{t}}\right)}{p\left(A_{\mathrm{t}}\right)} \geq p\left(A_{\mathrm{t}}\right)
$$

Changing equation 3.43 , it is

$$
p\left(b_{\mathrm{t}}\right)-p\left(A_{\mathrm{t}}\right) \geq-\frac{p\left(a_{\mathrm{t}}\right)}{p\left(A_{\mathrm{t}}\right)}
$$

Adding +1 to equation 3.44 , it is

$$
\underbrace{\boldsymbol{p}\left(\boldsymbol{b}_{\mathrm{t}}\right)+\mathbf{1}-\boldsymbol{p}\left(\boldsymbol{A}_{\mathrm{t}}\right)}_{\text {Exclusion }} \geq+1-\frac{p\left(a_{\mathrm{t}}\right)}{p\left(A_{\mathrm{t}}\right)}
$$

According to equation 2.33, the 1913 Henry Maurice Sheffer (1882-1964) stroke(Nicod, 1917, Sheffer, 1913) or equation 3.45 becomes

$$
p\left(A_{\mathrm{t}} \mid B_{\mathrm{t}}\right) \equiv p\left(A_{\mathrm{t}} \uparrow B_{\mathrm{t}}\right) \equiv \underbrace{\boldsymbol{p}\left(\boldsymbol{b}_{\mathrm{t}}\right)+\mathbf{1}-\boldsymbol{p}\left(\boldsymbol{A}_{\mathrm{t}}\right)}_{\text {Exclusion }} \geq+1-\frac{p\left(a_{\mathrm{t}}\right)}{p\left(A_{\mathrm{t}}\right)}
$$


In other words, a rough and an approximate estimate of the mutually exclusive relationship is given by the equation

$$
p\left(A_{\mathrm{t}} \mid B_{\mathrm{t}}\right) \geq+1-\frac{p\left(a_{\mathrm{t}}\right)}{p\left(A_{\mathrm{t}}\right)}
$$

In reality, the mutually exclusive relationship $+p\left(A_{\mathrm{t}} \mid B_{\mathrm{t}}\right)$ is much stronger than estimated by the relationship $+1-\frac{p\left(a_{\mathrm{t}}\right)}{p\left(A_{\mathrm{t}}\right)}$. At the end, it is $p\left(A_{\mathrm{t}} \mid B_{\mathrm{t}}\right) \geq+1-\frac{p\left(a_{\mathrm{t}}\right)}{p\left(A_{\mathrm{t}}\right)}$. Many times, the efficacy of treatment or prevention interventions is judged by randomized, placebo $23,24,25$ controlled trials (PCT). A key point of view of PCT is the use of placebo controls in trials, even under conditions with no effective treatment. However, withholding a treatment might pose negligible risks or even serious harm to participants of a placebo group. An inappropriate placebo group in PCT increases the cost and might induce study design caused bias, and therefore requires more than ethical justification. Mathematically, equation 3.47 or

$$
p\left(A_{\mathrm{t}} \mid B_{\mathrm{t}}\right) \geq+1-\frac{p\left(a_{\mathrm{t}}\right)}{p\left(A_{\mathrm{t}}\right)}
$$

offers the possibility to test the efficacy of a drug or a treatment even without a placebo group.

Example.

The 1995 West of Scotland Coronary Prevention Study(see Barukčić, 2019d), also known as WOSCOPS $^{26}$, compared $40 \mathrm{mg}$ pravastatin to placebo. In about 3302 men aged 45-64 years used $40 \mathrm{mg}$ pravastatin while 106 of these participants died due to any cause. Table 16 might provide a review of the data of this study.

Table 16. Pravastatin $40 \mathrm{mg}$ and death (WOSCOPS Study, 1995).

\begin{tabular}{lllll}
\hline & \multicolumn{4}{c}{ Death } \\
& & YES & NO \\
\hline Pravastatin 40 mg & YES & 106 & 3196 & 3302 \\
& NO & 135 & 3158 & 3293 \\
\hline & & 241 & 6354 & 6595
\end{tabular}

Independent of any study design, $40 \mathrm{mg}$ pravastatin excludes death due to any cause in men aged 45-64 years with the probability

$$
p\left(A_{\mathrm{t}} \mid B_{\mathrm{t}}\right) \geq+1-\frac{p\left(a_{\mathrm{t}}\right)}{p\left(A_{\mathrm{t}}\right)}=+1-\frac{21}{3302}=0,9934721790
$$

which indicates a positive result. In reality, the relationship is better than this estimation (see equation 3.49). In 1990 Germany, the death rate in men aged 45-64 years has been about 0.3 to $2 \%{ }^{27}$. In

\footnotetext{
${ }^{23}$ PMID: 24035802

${ }^{24}$ PMID: 29510711

${ }^{25}$ PMID: 27703733

${ }^{26}$ WOSCOPS study, 1995

${ }^{27}$ Wikipedia, Mortality in Germany 1990 (before use of pravastatin)
} 
Germany 1990, under normal circumstances, about 10 up to 66 of 3302 men aged 45-64 years would have died independent of any use of the drug pravastatin. A use of the drug pravastatin should improve this situation. During the trial period (average follow-up period was 4.9 years) 106 pravastatin participants died. In other words, per year $(((106 / 4,9)=21) / 3302) * 100=0,655137888 \%$ pravastatin participants died. However, the evidence of a decreased death rate in the group of patients treated with pravastatin compared to the death rate of the population of men aged $45-64$ years $(0,655137888 \%$ v.s 0.3 to $2 \%$ ) does not follow for sure. The relative risk reduction (per year) can be calculated as

$$
\begin{aligned}
\operatorname{RRR}(\text { Pravastatin } 40 m g, \text { Death }(\text { Per Year })) & \equiv\left(1-\left(\frac{a_{\mathrm{t}} \times \underline{A}_{\mathrm{t}}}{A_{\mathrm{t}} \times c_{\mathrm{t}}}\right)\right) \times 100 \\
& \equiv\left(1-\left(\frac{21 \times 3185}{3217 \times 27}\right)\right) \times 100 \\
& \equiv 22,99588989
\end{aligned}
$$

or as $22,99588989 \%$. However, whether a therapy with $40 \mathrm{mg}$ pravastatin and to what extent is really of any benefit for men aged 45-64 years remains to be proven. Pravastatin posses anti-viral (human cytomegalovirus) effects. The WOSCOPS study did not answer the question whether the lowering of the blood cholesterol levels or the control of a human cytomegalovirus infection reduces the risk of coronary heart disease or both or none. We still have to gain some experience with the use of the exclusion relationship. Nonetheless, the example before clearly demonstrates the dangers emanating from unsuitable statistical methods and the importance of a logically consistent study design. Otherwise, we run the risk of reaching conclusions which have nothing or too little in common with objective reality, while humans will suffer unnecessary harm.

\subsection{Mutually exclusive events and study design}

A study design has different tasks and functions. It should be noted, however, that regardless of whether the data are achieved by a placebo controlled randomized trial or a case-control study design, data need to be provided by the studies which enable us to draw the same conclusions. Therefore, the reduction of study design bias is of far-reaching and extraordinary importance.

Theorem 3.7. In general, a study design of a study which investigates an exclusion relationship between the events $A_{t}$ and $B_{t}$ should assure as much as possible (see equation 2.61) an index of independence (Barukčić, 2019a) given as

$$
\operatorname{IOI}\left(A_{t}, B_{t}\right) \equiv \frac{A_{t}+\underline{B}_{t}}{N}-1 \equiv 0
$$

Proof by direct proof. The premise

$$
+1 \equiv+1
$$

is true. In the following, we rearrange the premise. We obtain

$$
p\left(A_{\mathrm{t}} \mid B_{\mathrm{t}}\right) \equiv p\left(A_{\mathrm{t}} \mid B_{\mathrm{t}}\right)
$$

In the following, we would like to analyse this relationship independent of any type of study. From the point of view of a placebo controlled randomized trial (see equation 3.28), this relationship is given 
approximately as

$$
p\left(A_{\mathrm{t}} \mid B_{\mathrm{t}}\right) \equiv 1-\frac{p\left(a_{\mathrm{t}}\right)}{p\left(A_{\mathrm{t}}\right)}
$$

Ideally, the type of study should not have any influence on the conclusions drawn. From the point of view of a case control study, it is (see equation 3.36)

$$
1-\frac{p\left(a_{\mathrm{t}}\right)}{p\left(B_{\mathrm{t}}\right)} \equiv 1-\frac{p\left(a_{\mathrm{t}}\right)}{p\left(A_{\mathrm{t}}\right)}
$$

or

or

$$
\frac{p\left(a_{\mathrm{t}}\right)}{p\left(B_{\mathrm{t}}\right)} \equiv \frac{p\left(a_{\mathrm{t}}\right)}{p\left(A_{\mathrm{t}}\right)}
$$

and at the end

$$
\frac{1}{p\left(B_{\mathrm{t}}\right)} \equiv \frac{1}{p\left(A_{\mathrm{t}}\right)}
$$

$$
p\left(A_{\mathrm{t}}\right) \equiv p\left(B_{\mathrm{t}}\right)
$$

Equation 3.58 becomes

$$
p\left(A_{\mathrm{t}}\right) \equiv 1-p\left(\underline{B}_{\mathrm{t}}\right)
$$

Multiplying equation 3.59 by the sample size $\mathrm{N}$, it is

$$
\left(N \times p\left(A_{\mathrm{t}}\right)\right) \equiv N-\left(N \times p\left(\underline{B}_{\mathrm{t}}\right)\right)
$$

Equation 3.60 becomes

$$
A_{\mathrm{t}} \equiv N-\underline{B}_{\mathrm{t}}
$$

and

$$
A_{\mathrm{t}}+\underline{B}_{\mathrm{t}} \equiv N
$$

Dividing equation by the sample size $\mathrm{N}$, it is

$$
\frac{A_{\mathrm{t}}}{N}+\frac{B_{\mathrm{t}}}{N} \equiv \frac{N}{N} \equiv+1
$$

Rearranging equation 3.63 , it is

$$
\frac{A_{\mathrm{t}}+\underline{B}_{\mathrm{t}}}{N}-1 \equiv 0
$$

The study design of a study which investigates an exclusion relationship between the events $A_{t}$ and $\mathrm{B}_{\mathrm{t}}$ should assure as much as possible (see equation 2.61) an index of independence(Barukčić, 2019a) given as

$$
I O I\left(A_{\mathrm{t}}, B_{\mathrm{t}}\right) \equiv \frac{A_{\mathrm{t}}+\underline{B}_{\mathrm{t}}}{N}-1 \equiv 0
$$

Assumed that certain condition like the one detailed above cannot be met, there is a risk of completely unworldly conclusions, which could be without any meaning or understanding. A consequence of equation 3.65 is the need for the study design to assure conditions as much as possible where

$$
b_{\mathrm{t}} \equiv c_{\mathrm{t}}
$$




\subsection{Moderna}

How effective is Moderna's Covid-19 vaccine in preventing COVID-19 deaths? Grange et al. (see Grange et al., 2021) investigated COVID-19-related deaths in Scotland with respect to Moderna's Covid-19 vaccine.The data and the statistical analysis is presented by table 2. As can be seen, an IOR $=-1$ indicates correctly that an exclusion relationship is given. This position is supported by the relative risk $R R=0$, which indicates, that an exclusion relationship is given. The causal relationship $\mathrm{k}$ is negative and significant and demands an exclusion relationship. The index of independence with $\mathrm{p}(\mathrm{IOI})=0,012604762$ allows some conclusions about an exclusion relationship between Moderna's Covid-19 vaccine and Covid-19 death. In other words, Moderna's Covid-19 vaccine is preventing very effectively against COVID-19 death. The Moderna vaccine efficacy (Greenwood and Yule, 1915) can be calculated as

$$
\begin{aligned}
V E(\text { Moderna vaccine, Covid-19death }) & \equiv\left(1-\left(\frac{a_{\mathrm{t}} \times \underline{A_{\mathrm{t}}}}{A_{\mathrm{t}} \times c_{\mathrm{t}}}\right)\right) \times 100 \\
& \equiv\left(1-\left(\frac{0 \times 3231870}{41496 \times 236}\right)\right) \times 100 \\
& \equiv 100
\end{aligned}
$$

or as $100 \%$. 


\subsection{BionTech}

How effective is BionTech/Pfizer's Covid-19 vaccine in preventing COVID-19 deaths? Grange et al. (see Grange et al., 2021) investigated COVID-19-related deaths in Scotland with respect to BionTech/Pfizer's Covid-19 vaccine Covid-19 vaccine. The data and the statistical analysis is presented by table 3. Based on the data of the study of Grange et al. (see Grange et al., 2021), the probability of an exclusion relationship (see table 3 ) has been calculated very conservatively, approximately as $\mathrm{p}=$ 0,9999623103. Let us assume that the rest of Scotland's population (about 4090424 inhabitants) is not vaccinated against Covid-19 while about 1 to $2 \%$ would die due to Covid-19 virus infection (see table 17).

Table 17. BionTech vaccine and Covid-19 death (Study Grange et al. , 2021 ).

\begin{tabular}{lcccc}
\hline & \multicolumn{4}{c}{ Covid-19 death } \\
& & YES & NO \\
\hline BionTech vaccine & YES & 47 & 1246979 & 1247026 \\
& NO & 48803 & 4041621 & 4090424 \\
\hline & & 48850 & 5288600 & 5337450
\end{tabular}

Under these assumptions, the BionTech vaccine efficacy (Greenwood and Yule, 1915) can be calculated as

$$
\begin{aligned}
V E(\text { BionTech vaccine, Covid-19death }) & \equiv\left(1-\left(\frac{a_{\mathrm{t}} \times \underline{A}_{\mathrm{t}}}{A_{\mathrm{t}} \times c_{\mathrm{t}}}\right)\right) \times 100 \\
& \equiv\left(1-\left(\frac{47 \times 4090424}{1247026 \times 48803}\right)\right) \times 100 \\
& \equiv 99,68410398
\end{aligned}
$$

or as $99,68410398 \%$.

In Germany about 83129285 inhabitants are living. If all inhabitants of Germany were vaccinated by the BioNTech vaccine, then less than $(1-0,9999623103) * 83129285=3133$ inhabitants would die because of Covid-19. 
3.9. ... and again AstraZeneca Covid-19 vaccine

Let us assume that the rest of Scotland's population (about 3288252 inhabitants) is not vaccinated against Covid-19 while about 1 to $2 \%$ of these inhabitants would die due to Covid-19 virus infection (see table 18).

Table 18. AstraZeneca vaccine and Covid-19 death (Study Grange et al. , 2021 ).

\begin{tabular}{lcccc}
\hline & \multicolumn{4}{c}{ Covid-19 death } \\
& & YES & NO \\
\hline AstraZeneca vaccine & YES & 188 & 2026010 & 2026198 \\
& NO & 48662 & 3239590 & 3288252 \\
\hline & & 48850 & 5265600 & 5314450
\end{tabular}

Under these assumptions, the AstraZeneca vaccine vaccine efficacy (Greenwood and Yule, 1915) can be calculated as

$$
\begin{aligned}
V E(\text { AstraZeneca vaccine, Covid }-19 \text { death }) & \equiv\left(1-\left(\frac{a_{\mathrm{t}} \times \underline{A}_{\mathrm{t}}}{A_{\mathrm{t}} \times c_{\mathrm{t}}}\right)\right) \times 100 \\
& \equiv\left(1-\left(\frac{188 \times 3288252}{2026198 \times 48662}\right)\right) \times 100 \\
& \equiv 99,37302373
\end{aligned}
$$

or as $99,37302373 \%$. Contrary to discussion, the AstraZeneca vaccine itself is highly effective. In reality, more than about 1 to $2 \%$ of these inhabitants not vaccinated against a Covid-19 infection will die due to Covid-19 with the consequence that AstraZeneca's vaccine efficacy is much better than the calculated $(99,37302373 \%)$ one. However, as can be seen (see table 4) the study design, which is completely without any sense $(\mathrm{p}(\mathrm{IOI})=0,618923151 ; \mathrm{p}(\mathrm{IOU})=0,380932655)$ can lead to erroneous conclusions. Looking very closely, based on this study design, we would have to accept the hypothesis that AstraZeneca Covid-19 vaccine has been the cause of the Covid-19 death of the people vaccinated.

Reasons.

The causal relationship is positive, the risk ratio $\mathrm{RR}$ is $\mathrm{RR}>1$, IOR is not negative et cetera. In toto, the data of Grange et al. (see Grange et al., 2021) demand us to accept the Null-hypothesis: without vaccination with AstraZeneca Covid-19 no Covid-19 death ( $\mathrm{p}$ (SINE)=0,9999853362, $\mathrm{p}$ Value $(\mathrm{SINE})=0,0000146637)$. Such a conclusion is of course beyond any conceivable logic. Unfortunately, the data of the study of Grange et al. (see Grange et al., 2021) do support and require such a conclusion. Therefore, where is the error?

Firstly.

The study design does not allow such a conclusion. A p (IOU) $=0,380932655$ it too high for such a sample size. The data presented should not be used for the analyses of conditio sine qua non et cetera.

Secondly.

Based on the data of the study of Grange et al. (see Grange et al., 2021), the probability of an exclusion relationship (see table 4) has been calculated very conservatively, approximately as $\mathrm{p}=$ 0,9999072154. In Germany about 83129285 inhabitants are living. If all inhabitants of Germany were 
vaccinated by AstraZeneca Covid-19 vaccine, then less than $(1-0,9999072154) * 83129285=7713$ inhabitants would die because of Covid-19. In contrast to these 7713 cases calculated according to the data of the study of Grange et al. (see Grange et al., 2021), in Germany today (December 1, 2021) more than 100000 thousand of inhabitants died because of Covid-19 infection. This supports a vaccine efficacy of AstraZeneca's Covid-19 vaccine of about $((7713 / 56007713) /(92287 / 27121572)) * 100$ $=95,953 \%$. A critic may note that these 7713 patients died because of the AstraZeneca Covid-19 vaccination and not because of the Covid-19 virus infection. However, this topic has been discussed to the negative in great detail somewhere elsewhere.

Thirdly.

Great care and caution is required when analysing data. As can be seen from the table 4 and table 5, seriously wrong conclusions can follow if statistical methods are blindly applied to a data set without any meaning and without any understanding. Before data can be re-analysed, great attention should be paid to the facts, whether these data allow us at all to analyse the same. What is the design of the study, et cetera?

\section{Discussion}

Logically consistent statistical methods alone are not sufficient to reliably and automatically recognize conditions or cause-effect relationships, et cetera. In this context, a very careful assessment of the quality of the data and an evaluation of the quality of the study design of a study is of very great importance too. Nonetheless, to some extent, it is possible to rely on the index of unfairness(Barukčić, 2019b) and the index of independence(Barukčić, 2019a). However, additional tools are necessary in order to help scientist to improve the quality of scientific publications.

\section{Conclusion}

Mutually exclusive events can be recognized with a probability bordering on certainty.

\section{Acknowledgments}

No funding or any financial support by a third party was received.

\section{Patient consent for publication}

Not required.

\section{Conflict of interest statement}

No conflict of interest to declare.

\section{References}

A. J. Ayer. Negation. The Journal of Philosophy, 49(26):797-815, January 1952. doi: 10.2307/ 2020959. JSTOR. 
Monya Baker. 1,500 scientists lift the lid on reproducibility. Nature, 533(7604):452-454, 52016. ISSN 1476-4687. doi: 10.1038/533452a. PMID: 27225100.

Ilija Barukčić. Die Kausalität. Wiss.-Verl., Hamburg, 1. aufl. edition, January 1989. ISBN 3-98022160-1. ISBN: 978-3-9802216-0-1.

Ilija Barukčić. Die Kausalität. Scientia, Wilhelmshaven, 2., völlig überarb. aufl. edition, January 1997. ISBN 3-9802216-4-4. ISBN: 978-3-9802216-4-1.

Ilija Barukčić. Causality: New statistical methods. Books on Demand GmbH, Norderstedt, Germany, January 2005. ISBN 978-3-8334-3645-1.

Ilija Barukčić. Anti Heisenberg-Refutation Of Heisenberg's Uncertainty Relation. In American Institute of Physics - Conference Proceedings, volume 1327, pages 322-325, Linnaeus University, Växjö, Sweden, June 14 - 17, 2010, January 2011a. doi: 10.1063/1.3567453. AQT. Web of Science. Full text: American Institute of Physics.

Ilija Barukčić. The Equivalence of Time and Gravitational Field. Physics Procedia, 22:56-62, January 2011b. ISSN 18753892. doi: 10.1016/j.phpro.2011.11.008. ICPST 2011. Web of Science. Free full text: Elsevier.

Ilija Barukčić. Anti-Bell - Refutation of Bell's theorem: In: Quantum Theory: Reconsideration of Foundations-6 (QTRF6), Växjö, (Sweden), 11-14 June 2012. In American Institute of Physics Conference Proceedings, volume 1508, pages 354-358, Växjo, Sweden, 2012. American Institute of Physics - Conference Proceedings. doi: 10.1063/1.4773147. QTRF 6. Web of Science. Full text: American Institute of Physics.

Ilija Barukčić. The Mathematical Formula of the Causal Relationship k. International Journal of Applied Physics and Mathematics, 6(2):45-65, January 2016. doi: 10.17706/ijapm.2016.6.2.45-65. IJAPM. Free full text: IAP.

Ilija Barukčić. Die Kausalität (1989). Books on Demand, Norderstedt, reprint edition, 2017a. ISBN 978-3-7448-1595-6. ISBN-13: 9783744815956.

Ilija Barukčić. Theoriae causalitatis principia mathematica. Books on Demand, Norderstedt, $2017 \mathrm{~b}$. ISBN 978-3-7448-1593-2. ISBN-13: 9783754331347.

Ilija Barukčić. Index of Independence. Modern Health Science, 2(2):1-25, 10 2019a. ISSN 2576-7305. doi: 10.30560/mhs.v2n2p1. URL https://j.ideasspread.org/index.php/mhs/article/ view/331. Modern Health Science.

Ilija Barukčić. Index of Unfairness. Modern Health Science, 2(1):22, 4 2019b. ISSN 2576-7305, 2576-7291. doi: 10.30560/mhs.v2n1p22. Modern Health Science.

Ilija Barukčić. The P Value of likely extreme events. International Journal of Current Science Research, 5(11):1841-1861, 2019c. IJCSR . Free full text: ZENODO.

Ilija Barukčić. Statins and death due to any cause -all doubts removed? December 2019d. doi: 10.5281/zenodo.3902771. URL https://doi .org/10.5281/zenodo. 3902771. PubMed NLM ID: 101671355. 
Ilija Barukčić. Causal relationship k. International Journal of Mathematics Trends and Technology IJMTT, 66(10):76-115, 2020a. URL http://www.ijmttjournal.org/archive/ ijmtt-v66i10p512. Publisher: Seventh Sense Research Group SSRG.

Ilija Barukčić. Locality and Non locality. European Journal of Applied Physics, 2(5):1-13, October 2020b. ISSN 2684-4451. doi: 10.24018/ejphysics.2020.2.5.22. URL https: //ej-physics . org/ index.php/ejphysics/article/view/22. Free full text: EJAP.

Ilija Barukčić. N-th index D-dimensional Einstein gravitational field equations. Geometry unchained., volume 1. Books on Demand GmbH, Hamburg-Norderstedt, 1 edition, 2020c. ISBN 978-3-75264490-6. ISBN-13: 9783752644906 . Free full text (preprint): ZENODO.

Ilija Barukčić. Zero and infinity. Mathematics without frontiers. Books on Demand GmbH, HamburgNorderstedt, 1 edition, 2020d. ISBN 978-3-7519-1873-2. ISBN-13: 9783751918732.

Ilija Barukčić. Index of relationship. Causation, 16(8):5-37, April 2021a. doi: 10.5281/zenodo. 5163179. Free full text: Zenodo.

Ilija Barukčić. The causal relationship k. MATEC Web of Conferences, 336:09032, 2021b. ISSN 2261-236X. doi: 10.1051/matecconf/202133609032. CSCNS2020. Web of Science. Free full text: EDP Sciences.

Ilija Barukčić. The logical content of the risk ratio. Causation, 16(4):5-41, February 2021c. doi: 10.5281/zenodo.4679509. Free full text: Zenodo.

Ilija Barukčić and Okoh Ufuoma. Analysis of Switching Resistive Circuits A Method Based on the Unification of Boolean and Ordinary Algebras. Books on Demand, Norderstedt, first edition edition, 2020. ISBN 978-3-7519-8474-4. ISBN-13: 9783751984744.

Jacobi Bernoulli. Ars conjectandi, Opus posthumus: Accedit Tractatus de seriebus infinitis ; et epistola Gallice scripta De Ludo Pilae Reticularis. Impensis Thurnisiorum [Tournes], fratrum, Basileae (Basel, Suisse), January 1713. doi: 10.3931/e-rara-9001. Free full text: e-rara, Zurich, CH.

Raffaele Bombelli. L'algebra : opera di Rafael Bombelli da Bologna, divisa in tre libri : con la quale ciascuno da se potrà venire in perfetta cognitione della teorica dell'Aritmetica : con una tavola copiosa delle materie, che in essa si contengono. per Giovanni Rossi, Bolgna (Italy), 1579. Free full text: e-rara, Zurich, $\mathrm{CH}$.

George Boole. An investigation of the laws of thought, on which are founded mathematical theories of logic and probabilities. New York, Dover, 1854. Free full text: archive.org, San Francisco, CA 94118, USA.

R. J. Cook and D. L. Sackett. The number needed to treat: a clinically useful measure of treatment effect. BMJ (Clinical research ed.), 310(6977):452-454, 2 1995. ISSN 0959-8138. doi: 10.1136/ bmj.310.6977.452. PMID: 7873954. Free full text: PMCID: PMC2548824.

J. Cornfield. A method of estimating comparative rates from clinical data; applications to cancer of the lung, breast, and cervix. Journal of the National Cancer Institute, 11(6):1269-1275, 6 1951. ISSN 0027-8874. PMID: 14861651. 
Roger Cotes and Edmond Halley. Logometria. Philosophical Transactions of the Royal Society of London, 29(338):5-45, January 1714. doi: https://doi.org/10.1098/rstl.1714.0002. URL https: //doi .org/10.1098/rstl.1714.0002. Publisher: Royal Society.

D. R. Cox. The regression analysis of binary sequences. Journal of the Royal Statistical Society. Series B (Methodological), 20(2):215-242, 1958. ISSN 0035-9246. JSTOR.

Leonhard Euler. Introductio in analysin infinitorum. apud Marcum-MIchaelem Bousquet \& socios, 1748. doi: 10.3931/e-rara-8740. Free full text: e-rara, Zurich, $\mathrm{CH}$.

Ronald Aylmer Fisher. On the Interpretation of Chi square from Contingency Tables, and the Calculation of P. Journal of the Royal Statistical Society, 85(1):87-94, 1922. ISSN 0952-8385. doi: 10.2307/2340521. JSTOR.

Ronald Aylmer Fisher. The logic of inductive inference. Journal of the Royal Statistical Society, 98 (1):39-82, 1935. ISSN 0952-8385. doi: 10.2307/2342435. JSTOR.

Eckart Förster and Yitzhak Y Melamed. "Omnis determinatio est negatio” - Determination, Negation and Self-Negation in Spinoza, Kant, and Hegel. In: Spinoza and German idealism. Eckart Forster $\mathcal{F}$ Yitzhak Y. Melamed (eds.). Cambridge University Press, Cambridge [England]; New York, 2012. ISBN 978-1-283-71468-6. URL https: //doi .org/10 . 1017/СB09781139135139.

H. T. Gonin. XIV. The use of factorial moments in the treatment of the hypergeometric distribution and in tests for regression. The London, Edinburgh, and Dublin Philosophical Magazine and Journal of Science, 21(139):215-226, January 1936. ISSN 1941-5982. doi: 10.1080/14786443608561573. Taylor and Francis.

William Sealy Gosset. The probable error of a mean. Biometrika, 6(1):1-25, 1908. ISSN 0006-3444. doi: 1. JSTOR.

William Sealy Gosset. The elimination of spurious correlation due to position in time or space. Biometrika, 10(1):179-180, 1914. ISSN 0006-3444. doi: 10.2307/2331746. JSTOR.

Zoe Grange, Audrey Buelo, Christopher Sullivan, Emily Moore, Utkarsh Agrawal, Khaled Boukhari, Iain McLaughlan, Diane Stockton, Colin McCowan, Chris Robertson, Aziz Sheikh, and Josephine L K Murray. Characteristics and risk of covid-19-related death in fully vaccinated people in scotland. Lancet (London, England), 398(10313):1799-1800, 11 2021. ISSN 0140-6736. doi: 10.1016/ S0140-6736(21)02316-3. PMCID: PMC8553268 PMID: 34756204.

Major Greenwood and G. Udny Yule. The statistics of anti-typhoid and anti-cholera inoculations, and the interpretation of such statistics in general. Proceedings of the Royal Society of Medicine, 8: 113-194, 6 1915. ISSN 0035-9157. doi: 10.1177/003591571500801433. PMID: 19978918. Free full text: PMCID: PMC2004181.

Klaus Hedwig. Negatio negationis: Problemgeschichtliche Aspekte einer Denkstruktur. Archiv für Begriffsgeschichte, 24(1):7-33, 1980. ISSN 0003-8946. URL www. jstor . org/stable/24359358.

F. H. Heinemann. The Meaning of Negation. Proceedings of the Aristotelian Society, 44:127-152, 1943. ISSN 0066-7374. URL www. jstor .org/stable/4544390. 
Friedrich Robert Helmert. Ueber die Wahrscheinlichkeit der Potenzsummen der Beobachtungsfehler und über einige damit im Zusammenhange stehende Fragen. Zeitschrift für Mathematik und Physik, 21(3):102-219, 1876.

Laurence R. Horn. A natural history of negation. University of Chicago Press, Chicago, 1989. ISBN 978-0-226-35337-1. URL https://emilkirkegaard.dk/en/wp-content/uploads/ A-natural-history-of-negation-Laurence-R.-Horn.pdf.

Christiaan Huygens and Frans van Schooten. De ratiociniis in ludo alae: In: Exercitationum mathematicarum liber primus [- quintus]. ex officina Johannis Elsevirii, Lugdunum Batavorum (Leiden, The Netherlands), January 1657. doi: 10.3931/e-rara-8813. Free full text: e-rara, Zurich, CH.

Michal Kicinski, David A. Springate, and Evangelos Kontopantelis. Publication bias in meta-analyses from the cochrane database of systematic reviews. Statistics in Medicine, 34(20):2781-2793, 9 2015. ISSN 1097-0258. doi: 10.1002/sim.6525. PMID: 25988604.

Mirjam J. Knol. Down with odds ratios: risk ratios in cohort studies and randomised clinical trials (article in dutch). Nederlands Tijdschrift Voor Geneeskunde, 156(28):A4775, 2012. ISSN 18768784. PMID: 22805792.

Anton Friedrich Koch. Die Selbstbeziehung der Negation in Hegels Logik. Zeitschrift für philosophische Forschung, 53(1):1-29, 1999. ISSN 0044-3301. URL www . jstor . org/stable/20484868.

A. Kolmogoroff. Grundbegriffe der Wahrscheinlichkeitsrechnung. Springer Berlin Heidelberg, Berlin, Heidelberg, January 1933. ISBN 978-3-642-49596-0. doi: 10.1007/978-3-642-49888-6.

Kenneth Kunen. Negation in logic programming. The Journal of Logic Programming, 4(4):289-308, December 1987. ISSN 0743-1066. doi: 10.1016/0743-1066(87)90007-0. URL http://www . sciencedirect.com/science/article/pii/0743106687900070.

Andreas Laupacis, David L. Sackett, and Robin S. Roberts. An assessment of clinically useful measures of the consequences of treatment. New England Journal of Medicine, 318(26):1728-1733, 1988. doi: 10.1056/NEJM198806303182605. PMID: 3374545. NEJM.

D. Massel and M. K. Cruickshank. The number remaining at risk: an adjunct to the number needed to treat. The Canadian Journal of Cardiology, 18(3):254-258, 3 2002. ISSN 0828-282X. PMID: 11907613.

Abraham de Moivre. The Doctrine of Chances or a Method of Calculating the Probability of Events in Play. printed by W. Pearson for the author, London, January 1718. doi: 10.3931/e-rara-10420. Free full text: e-rara, Zurich, $\mathrm{CH}$.

Russell Newstadt. Omnis Determinatio est Negatio: A Genealogy and Defense of the Hegelian Conception of Negation. Loyola University Chicago, Chicago (IL), dissertation edition, 2015. Free full text: Loyola University Chicago, USA.

Jerzy Neyman and Egon Sharpe Pearson. IX. On the problem of the most efficient tests of statistical hypotheses. Philosophical Transactions of the Royal Society of London. Series A, Containing Papers of a Mathematical or Physical Character, 231(694-706):289-337, 2 1933. ISSN 0264-3952, 20539258. doi: 10.1098/rsta.1933.0009. The Royal Society, London, GB. 
Jean George Pierre Nicod. A reduction in the number of primitive propositions of logic. Proceedings of the Cambridge Philosophical Society, 19:32-41, 1917.

Jean George Pierre Nicod. Les relations des valeurs et les relations de sens en logique formelle. Revue de métaphysique et de morale, 31:467-480, 1924.

Luca Pacioli. Summa de arithmetica, geometria, proportioni et proportionalità. Unknown publisher, Venice, 1494. URL http://doi . org/10.3931/e-rara-9150. Free full text: e-rara, Zurich, CH.

Karl Pearson. XV. On certain properties of the hypergeometrical series, and on the fitting of such series to observation polygons in the theory of chance. The London, Edinburgh, and Dublin Philosophical Magazine and Journal of Science, 47(285):236-246, January 1899. ISSN 1941-5982. doi: 10.1080/ 14786449908621253. Taylor and Francis.

Karl Pearson. X. On the criterion that a given system of deviations from the probable in the case of a correlated system of variables is such that it can be reasonably supposed to have arisen from random sampling. The London, Edinburgh, and Dublin Philosophical Magazine and Journal of Science, 50(302):157-175, July 1900. ISSN 1941-5982. doi: 10.1080/14786440009463897. Taylor and Francis.

Karl Pearson. Mathematical contributions to the theory of evolution. XIII. On the theory of contingency and its relation to association and normal correlation. Biometric Series I. Dulau and Co., London, January 1904. Free full text: archive.org, San Francisco, CA 94118, USA.

Robert Recorde. The whetstone of witte, whiche is the seconde parte of Arithmetike: containyng thextraction of Rootes: The Coßike practise, with the rule of Equation: and the woorkes of Surde Nombers. Jhon Kyngstone, London, 1557. Free full text: archive.org, San Francisco, CA 94118, USA.

Michel Rolle. Traité d'algèbre ou principes généraux pour résoudre les questions de mathématique. chez Estienne Michallet, Paris (France), 1690. Free full text: e-rara, Zurich, CH.

Josiah Royce. Negation, volume 9 of Encyclopaedia of Religion and Ethics. J. Hastings (ed.). Charles Scribner's Sons, New York (USA), 1917. Free full text: archive.org, San Francisco, CA 94118, USA.

DL Sackett, JJ Deeks, and DG Altman. Sackett dl, deeks jj, altman dg. down with odds ratios! evidence-based med. 1996;1:164-166. Evidence-Based Med., 1:164-166, 1996.

D. A. Sadowsky, A. G. Gilliam, and J. Cornfield. The statistical association between smoking and carcinoma of the lung. Journal of the National Cancer Institute, 13(5):1237-1258, 4 1953. ISSN 0027-8874. PMID: 13035448.

Henry Maurice Sheffer. A set of five independent postulates for boolean algebras, with application to logical constants. Transactions of the American Mathematical Society, 14(4):481-488, 1913. ISSN 0002-9947, 1088-6850. doi: 10.1090/S0002-9947-1913-1500960-1. Free full text: archive.org, San Francisco, CA 94118, USA. 
J. L. Speranza and Laurence R. Horn. A brief history of negation. Journal of Applied Logic, 8(3): 277-301, September 2010. ISSN 1570-8683. doi: 10.1016/j.jal.2010.04.001. URL http://www . sciencedirect.com/science/article/pii/S1570868310000236.

Alfred Tennyson. Poems. In Two Volumes. Vol. II. Poems in two volumes, published in 1842 (volume 2) by Alfred Tennyson. Edward Moxon, 1842. hrefhttps://archive.org/details/Tennysonpoems1842vol2/page/n103/mode/2upArchive.

J. v. Uspensky. Introduction To Mathematical Probability. McGraw-Hill Company, New York (USA), 1937. Free full text: archive.org, San Francisco, CA 94118, USA.

Michael V. Wedin. Negation and quantification in aristotle. History and Philosophy of Logic, 11 (2):131-150, January 1990. ISSN 0144-5340. doi: 10.1080/01445349008837163. URL https: //doi .org/10.1080/01445349008837163.

Johannes Widmann. Behende und hübsche Rechnung auff allen Kauffmanschafften. Conrad Kachelofen, Leipzig (Holy Roman Empire), 1489. Bayerische Staatsbibliothek .

Robin J. Wilson. Euler's pioneering equation: the most beautiful theorem in mathematics. Oxford University Press, Oxford, United Kingdom, first edition edition, 2018. ISBN 978-0-19-879492-9. OCLC: ocn990970269.

Frank Yates. Contingency Tables Involving Small Numbers and the Chi square Test. Supplement to the Journal of the Royal Statistical Society, 1(2):217-235, 1934. ISSN 1466-6162. doi: 10.2307/ 2983604. JSTOR.

George Udny Yule. On the methods of measuring association between two attributes. Journal of the Royal Statistical Society, 75(6):579-652, 1912. ISSN 0952-8385. doi: 10.2307/2340126. JSTOR.

George Udny Yule and Karl Pearson. Vii. on the association of attributes in statistics: with illustrations from the material of the childhood society, \&c. Philosophical Transactions of the Royal Society of London. Series A, Containing Papers of a Mathematical or Physical Character, 194(252-261): 257-319, 1 1900a. doi: 10.1098/rsta.1900.0019.

George Udny Yule and Karl Pearson. VII. On the association of attributes in statistics: with illustrations from the material of the childhood society. Philosophical Transactions of the Royal Society of London. Series A, Containing Papers of a Mathematical or Physical Character, 194(252-261): 257-319, 1 1900b. doi: 10.1098/rsta.1900.0019. The Royal Society, London, GB. 
(C) 2021 Ilija Barukčić ${ }^{a}, b$

, $c, d, e, f, g, h, i, j, k, l, m, n$, Jever, Germany, December 1, 2021. All rights reserved. Alle Rechter vorbehalte. This is an open access article which can be downloaded under the terms of the Creative Commons Attribution License (http://creativecommons.org/licenses/by/4.0).

I was born October, $1^{\text {st }} 1961$ in Novo Selo, Bosnia and Herzegovina, former Yogoslavia. I am of Croatian origin. From 1982-1989 C.E., I studied human medicine at the University of Hamburg, Germany. Meanwhile, I am working as a specialist of internal medicine. My basic field of research since my high school days at the Wirtschaftsgymnasium Bruchsal, Baden Württemberg, Germany is the mathematization of the relationship between a cause and an effect valid without any restriction under any circumstances including the conditions of classical logic, probability theory, quantum mechanics, special and general theory of relativity, human medicine et cetera. I endeavour to investigate positions of quantum mechanics, relativity theory, mathematics et cetera, only insofar as these positions put into question or endanger the general validity of the principle of causality.

\footnotetext{
${ }^{a}$ https: //orcid .org/OQOQ-Q0Q2-6988-2780

${ }^{b}$ https://cel. webofknowledge.com/InboundService.do?app=wos\& product $=$ CEL\&Func $=$ Frame\&SrcApp $=$ Publons\&SrcAuth $=$ Publons_CEL\& locale=en-US\&SID=F4r5Tsr30crmFbYrqiF\&customersID=Publons_CEL\& smartRedirect=yes\&mode=FullRecord\&IsProductCode=Yes\&Init=Yes\& action=retrieve\&UT $=$ WOS\%3A000298855300006

${ }^{c}$ https://publons.com/researcher/3501739/ilija-barukcic/

${ }^{d}$ https://www. scopus. com/authid/detail .uri?authorId= 37099674500

${ }^{e}$ https://WwW. scopus. com/authid/detail. uri?authorId= 54974181600

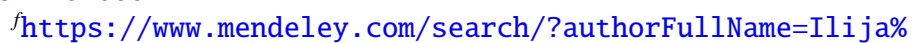
20Baruk\%C4\%8Di\%C4\%87\&page=1\&query=Barukcic\&sortBy=relevance ${ }^{g}$ https://www . researchgate . net/profile/Ilija-Barukcic- 2

${ }^{h}$ https : / / zenodo . org/search?page=1\&size=20\&q=keywords : \%22Baruk\%C4\%8Di\%C4\%87\%22\&sort=mostviewed ${ }^{i}$ https : / / zenodo org/search?page=1\&si ze=20\&q=keywords : \%22Baruk\%C4\%8Di\%C4\%87, \%20Conference\%22

${ }^{j}$ https://twitter. com/ilijabarukcic?lang=de

${ }^{k}$ https://twitter.com/Causation_Journ

${ }^{l}$ https://vixra.org/author/ilija_barukcic

${ }^{m}$ https://www . youtube. com/channel/UCwf3w1IngcukI00jpw8HTwg

${ }^{n}$ https://portal .dnb.de/opac/showNextResultSite? currentResultId=\%22Barukcic\%22\%26any\&currentPosition=30
} 